\title{
Cubic M-polar Fuzzy Hybrid Aggregation Operators with Dombi's T-norm and T-conorm with Application
}

\author{
Muhammad Riaz ${ }^{1}\left(\mathbb{0}\right.$, Muhammad Abdullah Khokhar ${ }^{1}$, Dragan Pamucar ${ }^{2, * \mathbb{C}}$ and Muhammad Aslam ${ }^{3}$ \\ 1 Department of Mathematics, University of the Punjab, Lahore 54590, Pakistan; mriaz.math@pu.edu.pk (M.R.); \\ mabdullahk1394@gmail.com (M.A.K.) \\ 2 Department of Logistics, Military Academy, University of Defence in Belgarde, Belgarde 11000, Serbia \\ 3 Department of Mathematics, College of Sciences, King Khalid University, Abha 61413, Saudi Arabia; \\ muamin@kku.edu.sa \\ * Correspondence: dragan.pamucar@va.mod.gov.rs
}

Citation: Riaz, M.; Khokhar, M.A.; Pamucar, D.; Aslam, M. Cubic M-polar Fuzzy Hybrid Aggregation Operators with Dombi's T-norm and T-conorm with Application. Symmetry 2021, 13, 646. https://doi.org/ $10.3390 /$ sym 13040646

Academic Editor: José Carlos R. Alcantud

Received: 24 March 2021

Accepted: 6 April 2021

Published: 11 April 2021

Publisher's Note: MDPI stays neutral with regard to jurisdictional claims in published maps and institutional affiliations.

Copyright: (C) 2021 by the authors. Licensee MDPI, Basel, Switzerland. This article is an open access article distributed under the terms and conditions of the Creative Commons Attribution (CC BY) license (https:// creativecommons.org/licenses/by/ $4.0 /)$.

\begin{abstract}
A cubic m-polar fuzzy set (CmPFS) is a new hybrid extension of cubic set (CS) and m-polar fuzzy set (mPFS). A CS comprises two parts; one part consists of a fuzzy interval (may sometimes be a fuzzy number) acting as membership grade (MG), and the second part consists of a fuzzy number acting as non-membership grade (NMG). An mPFS assigns $m$ number of MGs against each alternative in the universe of discourse. A CmPFS deals with single as well as multi-polar information in the cubic environment. In this article, we explore some new aspects and consequences of the CmPFS. We define score and accuracy functions to find the priorities of alternatives/objects in multi-criteria decision-making (MCDM). For this objective, some new operations, like addition, scalar/usual multiplication, and power, are defined under Dombi's t-norm and t-conorm. We develop several new aggregation operators (AOs) using cubic m-polar fuzzy Dombi's t-norm and t-conorm. We present certain properties of suggested operators like monotonicity, commutativity, idempotency, and boundedness. Additionally, to discuss the application of these AOs, we present an advanced superiority and inferiority ranking (SIR) technique to deal with the problem of conversion from a linear economy to a circular economy. Moreover, a comparison analysis of proposed methodology with some other existing methods is also given.
\end{abstract}

Keywords: cubic m-polar fuzzy set; Dombi's operations; cubic m-polar fuzzy aggregation operators with P-order (R-order); SIR technique; multi-criteria group decision making

\section{Introduction}

The three core concepts of conventional linear economy (CLE) are assemble, use, and dispose. This illustrates the acquisition of raw materials and their conversion into products that are ultimately discarded as waste. By depleting natural resources and adding toxins to the atmosphere, such waste generation causes environmental degradation. Natural resource extraction is inextricably linked to the so-called CLE [1-4]. The carcinogenic effects of human activities on the environment, such as water scarcity, soil depletion, greenhouse effect, and smog weather, are major global concerns. Climate change is, in reality, the most pressing problem we face. As a result, the United Nations Environment Programme (UNEP) has devised a broad definition of sustainable development, which is a concept that includes not only economic growth and environmental protection, but also social inclusion. Without a doubt, the CLE has aided humanity, but it has also been a big source of concern due to the challenges it poses. We are all aware that our common environment is insecure 
and unsustainable. We are aware of its complexities, such as waste generation, natural resource use, and biodiversity depletion, among others. Aside from these problems, we want to help our economies and provide opportunities to the world's growing population.

The ecological effects of CLE, while guaranteeing its benefits for humankind, cannot be avoided, but can be minimized to some extent. The minimizing effort of the adversities of CLE is referred as circular economy (CE) [1-4]. A CE is structured to recycle and regenerate goods, parts, and resources, allowing for a considerable difference between technological and biological processes at all periods of the recycling process. CE is not a new concept; rather, it is a modification of CLE, which guarantees a minor net impact on the climate. $\mathrm{CE}$ is intended to restore any harm to the resources while guaranteeing as little waste as possible during the entire life cycle of a good. A CE is an adjunct to a CLE, where resources are preserved as long as possible, and the optimum displacement is collected, retained, and regenerated at the end of each access lifespan. Many biochemical and geochemical cycles around the motivated the idea of circular economy. For instance, water evaporates from the earth water bodies, forms rain drops, comes back to the earth and again becomes a part of the rivers, seas, oceans etc. The idea of $\mathrm{CE}$ is being actively encouraged by many corporations and governments round the world.

Although businesses are agile and well-equipped, many people worldwide have attended series of conferences on sustainable practices, with discussions how well circular economy guidelines could be coordinated and applied. If we assume that a lack of creative business models would interfere with creating a sustainable future, it appears critical to identify more forward-thinking alternatives. In this regard, we see the circular economy as a modern way to practice sustainability that stems from the need for companies of all sizes to retain flexibility in order to meet these challenges. Despite the growing popularity of $\mathrm{CE}$ as a business model, there is still little formal empirical discussion in the literature on enterprise risk management. Financial/sustainable success is also seen as a priority over ecological, social, and ethical values in light of the numerous academic debates on sustainable and environmentally responsible businesses. As a result, the circular economy is a crucial and timely idea to investigate. The circular economy has captured the minds of elected officials and business leaders in order to help meet the overwhelming environmental goals. It is a practical way to improve asset flow efficiency and allocation of current supply and frameworks through material transfer, recycling, and conservation, with a focus on improving the effectiveness of existing performance measurement in businesses. Many scientists have worked tirelessly to develop mathematical models for solving CE decision problems in unpredictable environments. The readers are referred to the following papers for ore information [5-9].

\subsection{Literature Review}

There is an overwhelming amount of uncertain and vague information in a wide range of real scenarios. While dealing with real-life challenges such as decision-making, medical diagnosis, pattern recognition, sustainability, and many others, uncertainties play a significant role, and it is a challenging task for decision-makers to make sensible decisions while dealing with imperfect, uncertain, or vague data. In fact, the majority of the ideas we come across in our daily lives are ambiguous. In certain contexts, dealing with apprehension or confusion is a significant problem. Vagueness or ambiguity can be evident in a variety of ways, resulting in a wide range of concerns. As a result, there is a need to deal with the uncertainties. 
This idea was discussed by Zadeh [10] in 1965, who introduced a revolutionary idea of fuzzy set (FS) as a direct extension of crisp set. Researchers have introduced various theories and models to cope with the uncertainties in the real-life problems. Atanassov [11] introduced the intuitionistic fuzzy set (IFS), Molodtsov [12] originated the notion of a soft set (SS), Zhang $[13,14]$ presented the idea of bipolar fuzzy set (BFS), Smarandache $[15,16]$ proposed neutrosophic set, Cuong [17] introduced picture fuzzy set (PiFS), Yager [18,19] proposed Pythagorean fuzzy set (PyFS), and Yager [20] proposed q-rung orthopair fuzzy set (q-ROFS). These models have strong acceptance for modeling uncertainties in decisionmaking problems [21-25]. Dombi aggregation operators for information aggregation in the environment of different fuzzy sets have been studied by many researchers [26-32]. Chen et al. [33] introduced the idea of m-polar fuzzy sets to express multi-polarity in the objects/alternatives. Jun et al. [34] introduced cubic sets and their internal and external behaviors. Riaz and Hashmi [35] developed the notion of cubic m-polar fuzzy sets and established cubic m-polar fuzzy averaging aggregation operators for agribusiness MAGDM. Recently, Riaz and Hashmi [36-38] introduced some new extensions of fuzzy sets named as linear Diophantine fuzzy set (LDFS), soft rough linear Diophantine fuzzy set, and spherical linear Diophantine sets. Kamaci [39] introduced algebraic structure to LDFS with an interesting application to coding theory, which is based on LDFS codes.

Innovation of multi-criteria decision-making (MCDM) in fuzzy set theory is still an important topic at present. MCDM is a branch of decision science theory that is considered a cognitive based human behavior for choosing the best option under multiple criteria and has been widely applied across a variety of domains. One of the most difficult issues is to address uncertainties in MCDM by an efficient fuzzy model. Another objective in MCDM is to find ranking of feasible objects and then finally the selection of an optimal object. In actual decision-making, the individual needs to provide the assessment of the choices made by different types of assessment conditions, such as crisp numbers and intervals. However, in many situations, it is difficult for a person to opt for the correct option due to the existence of a variety of data inconsistencies that may occur due to lack of information or human error. Many aggregation operators (AOs) have been defined for information fusion [40-43]. Jain et al. [44] greatly contribution to circular economy by giving a DM solution in green marketing strategy.

\subsection{Objectives and Organization of the Paper}

The first objective of this paper is to address uncertainties more effectively by using cubic m-polar fuzzy numbers (CmPFNs). The second objective is to extend Dombi's operations to CmPFNs and develop various aggregation operators listed as follows. Cubic $\mathrm{m}$-polar fuzzy Dombi P-averaging operator (CmPFDPAO).Cubic m-polar fuzzy Dombi $\mathrm{R}$-averaging operator (CmPFDRAO). Cubic m-polar fuzzy Dombi weighted P-averaging operator (CmPFDWPAO). Cubic m-polar fuzzy Dombi weighted R-averaging operator (CmPFDWRAO). Cubic m-polar fuzzy Dombi ordered weighted P-averaging operator (CmPFDOWPAO). Cubic m-polar fuzzy Dombi ordered weighted R-averaging operator (CmPFDOWRAO). Cubic m-polar fuzzy Dombi hybrid P-averaging Operator ( $\mathrm{CmPFDH}-$ $P A O)$. Cubic m-polar fuzzy Dombi hybrid R-averaging operator (CmPFDHRAO). The third objective is to investigate certain properties of suggested operators like monotonicity, commutativity, idempotency, and boundedness. Additionally, proposed Dombi's AOs are more useful to investigate ranking of objects/alternatives in MCDM with the help of CmPFNs. The fourth objective to develop an advanced superiority and inferiority ranking 
(SIR) technique to deal with the problem of conversion from the linear economy to the circular economy.

The remainder of the paper is organized as follows. In Section 2, some basic concepts like fuzzy sets, m-polar fuzzy sets, and cubic sets are reviewed. In Section 3, we discuss some results of cubic m-polar fuzzy sets. In Section 4, we present some Dombi's operations for cubic m-polar fuzzy environment. In Section 5, some cubic m-polar fuzzy Dombi aggregation operators with P-order are defined. In Section 5, some cubic m-polar fuzzy Dombi aggregation operators with R-order are developed. An interesting application to the circular economy using the proposed operators is given in Section 7. An advanced superiority and inferiority ranking (SIR) technique to deal with the problem of conversion from the linear economy to the circular economy is developed in Section 7. Lastly, the conclusion of this research work is given in Section 8 .

\section{Preliminaries}

In this section, we review some basic concepts of fuzzy sets, m-polar fuzzy sets, and cubic sets.

Definition 1 ([10]). A fuzzy set in the universe of discourse $Q$ is defined as

$$
F=\left\{\left(\hbar, \mu_{F}(\hbar)\right): \hbar \in Q\right\}
$$

where the membership function is $\mu_{F}: Q \rightarrow[0,1]$ and the membership degree (MD) of $\hbar$ is $\mu_{F}(\hbar)$.

Definition 2 ([34]). A cubic set $\ddot{\mathbb{C}}$ on a universe $Q$ is an object of the form

$$
\ddot{\mathbb{C}}=\{(\hbar, A(\hbar), B(\hbar)): \hbar \in Q\}
$$

where $A(\hbar)$ is a fuzzy interval and $B(\hbar)$ is a fuzzy number assigned to the alternative $\hbar$ representing the membership and non-membership grades, respectively. For short, the cubic set can be denoted as $\langle A, B\rangle$.

Definition 3. An m-polar fuzzy set (mPFS) with universe $Q$ is a mapping, $Q \longrightarrow[0,1]^{m}$, that assigns m-independent fuzzy membership grades to each element of $Q$. An mPFS can be written as

$$
\mathfrak{M}_{\mathbb{P}}=\left\{\left\langle\gamma,\left(\mu_{i}(\gamma)\right)_{i=1}^{m}\right\rangle: \gamma \in Q\right\}
$$

Definition 4 ([35]). A cubic m-polar fuzzy set (CmPFS) in a universe $W$ is an object like $C_{c m}=\left\{\left(x,\left[\mu_{1}^{-}(x), \mu_{1}^{+}(x)\right],\left[\mu_{2}^{-}(x), \mu_{2}^{+}(x)\right], \cdots,\left[\mu_{m}^{-}(x), \mu_{m}^{+}(x)\right], \mu_{1}(x), \mu_{2}(x), \cdots, \mu_{m}(x)\right):\right.$ $x \in W\}$, where $\left[\mu_{j}^{-}(x), \mu_{j}^{+}(x)\right]$ are fuzzy intervals and $\mu_{j}(x)$ are fuzzy numbers. $\mu_{j}^{-}$are called lower fuzzy numbers and $\mu_{j}^{+}$are called upper fuzzy numbers. Briefly, we can write CmPFN as $\left(\left[\mu_{j}^{-}, \mu_{j}^{+}\right], \mu_{j}\right)_{j=1}^{m}$.

Definition 5 ([34]). Given two fuzzy intervals $J_{a}=\left[\mu_{a}^{-}, \mu_{a}^{+}\right]$and $J_{b}=\left[v_{b}^{-}, v_{b}^{+}\right]$, then

$1 \quad J_{a} \leq J_{b} \Leftrightarrow \mu_{a}^{-} \leq v_{b}^{-}$and $\mu_{a}^{+} \leq v_{b}^{+}$

$2 J_{a} \geq J_{b} \Leftrightarrow \mu_{a}^{-} \geq v_{b}^{-}$and $\mu_{a}^{+} \geq v_{b}^{+}$

$3 \quad J_{a}=J_{b} \Leftrightarrow \mu_{a}^{-}=v_{b}^{-}$and $\mu_{a}^{+}=v_{b}^{+}$

Definition 6 ([35]). Let $A=\left(\left[\mu_{j}^{-}, \mu_{j}^{+}\right], \mu_{j}\right)_{j=1}^{m}$ and $B=\left(\left[v_{j}^{-}, v_{j}^{+}\right], v_{j}\right)_{j=1}^{m}$, be the two CmPFNs.

$1 \quad$ (P-Order) $A \leq_{P} B \Leftrightarrow\left[\mu_{j}^{-}, \mu_{j}^{+}\right] \leq\left[v_{j}^{-}, v_{j}^{+}\right]$and $\mu_{j} \leq v_{j}$.

2 (R-Order) $A \leq_{R} B \Leftrightarrow\left[\mu_{j}^{-}, \mu_{j}^{+}\right] \leq\left[v_{j}^{-}, v_{j}^{+}\right]$and $\mu_{j} \geq v_{j}$. 
3 (Equality) $A=B \Leftrightarrow\left[\mu_{j}^{-}, \mu_{j}^{+}\right]=\left[v_{j}^{-}, v_{j}^{+}\right]$and $\mu_{j}=v_{j}$

for all $j=1,2, \cdots, m$.

\subsection{Operations for CmPFNs}

In this part we discuss some operations on CmPFNs (see [35]). Let $A_{i}=\left(\left[\mu_{i j}^{-}, \mu_{i j}^{+}\right], \mu_{i j}\right)_{j=1}^{m}$, $i \in \Omega$, be the collection of CmPFNs. Then

1 (Complement) $A_{i}^{c}=\left(\left[1-\mu_{i j}^{+}, 1-\mu_{i j}^{-}\right], 1-\mu_{i j}\right)_{j=1}^{m}$

2 (P-Maximum) $\vee_{P} A_{i}=\left(\left[\sup _{i \in \Omega} \mu_{i j}^{-}, \sup _{i \in \Omega} \mu_{i j}^{+}\right], \sup _{i \in \Omega} \mu_{i j}\right)_{j=1}^{m}$

3 (P-Minimum) $\wedge_{P} A_{i}=\left(\left[\inf _{i \in \Omega} \mu_{i j}^{-}, \inf _{i \in \Omega} \mu_{i j}^{+}\right], \inf _{i \in \Omega} \mu_{i j}\right)_{j=1}^{m}$

$4 \quad\left(\right.$ R-Maximum) $\vee_{P} A_{i}=\left(\left[\sup _{i \in \Omega} \mu_{i j}^{-}, \sup _{i \in \Omega} \mu_{i j}^{+}\right], \inf _{i \in \Omega} \mu_{i j}\right)_{j=1}^{m}$

$5 \quad$ (R-Minimum) $\wedge_{P} A_{i}=\left(\left[\inf _{i \in \Omega} \mu_{i j}^{-}, \inf _{i \in \Omega} \mu_{i j}^{+}\right], \sup _{i \in \Omega} \mu_{i j}\right)_{j=1}^{m}$

\section{Some Results on CmPFS}

In this section, we give some basic results of CmPFS that will help in the next section to better understanding of the proposed aggregation operators.

Definition 7. A CmPFS $\mathfrak{C}_{m}=\left\{\left\langle q,\left(\left[\mu_{j}^{-}(q), \mu_{j}^{+}(q)\right], \mu_{j}(q)\right)_{j=1}^{m}\right\rangle: q \in Q\right\}$ on a discourse $Q$ is said to be an Internal Cubic m-Polar Fuzzy Set (ICmPFS) if $\mu_{j}^{-}(q) \leq \mu_{j}(q) \leq \mu_{j}^{+}(q)$, for all $q \in Q$ and $j=1,2, \cdots, m$.

Definition 8. A CmPFS $\mathfrak{C}_{m}=\left\{\left\langle q,\left(\left[\mu_{j}^{-}(q), \mu_{j}^{+}(q)\right], \mu_{j}(q)\right)_{j=1}^{m}\right\rangle: q \in Q\right\}$ is referred to as External Cubic m-Polar Fuzzy Set (ECmPFS) if it is not internal, that is, if $\mu_{j}^{-}(q) \not \leq \mu_{j}(q) \not \leq$ $\mu_{j}^{+}(q)$, for some $q \in Q$ or $j=1,2, \cdots, m$.

Thus, ECmPFS is simply the negation of ICMPFS.

Definition 9. A CmPFS $\mathfrak{C}_{m}=\left\{\left\langle q,\left(A_{j}(q), \mu_{j}(q)\right)_{j=1}^{m}\right\rangle: q \in Q\right\}$ is characterized as a Null Cubic $m$-Polar Fuzzy Set (NCmPFS) if $A_{j}(q)=0$ and $\mu_{j}(q)=1$ for all $q \in Q$ and $j=1,2, \cdots, m$.

Definition 10. If for a CmPFS $\mathfrak{C}_{m}=\left\{\left\langle q,\left(A_{j}(q), \mu_{j}(q)\right)_{j=1}^{m}\right\rangle: q \in Q\right\}, A_{j}(q)=1$ and $\mu_{j}(q)=0$ for all $q \in Q$ and $j=1,2, \cdots, m$, it is called an Absolute Cubic m-Polar Fuzzy Set (ACmPFS).

Theorem 1. The set of all ICmPFSs on a discourse $Q$ is closed under the operation of complement; that is, $A$ is ICmPFS if and only if $A^{c}$ is ICmPFS.

Proof. Consider an Internal Cubic m-Polar Fuzzy Set $\mathfrak{C}_{m}=\left\{\left\langle q,\left(\left[\mu_{j}^{-}(q), \mu_{j}^{+}(q)\right], \mu_{j}(q)\right)_{j=1}^{m}\right\rangle\right.$ : $q \in Q\}$. Then $\mu_{j}^{-}(q) \leq \mu_{j}(q) \leq \mu_{j}^{+}(q)$, for all $q \in Q$ and $j=1,2, \cdots, m$. This implies that

$$
1-\mu_{j}^{+}(q) \leq 1-\mu_{j}(q) \leq 1-\mu_{j}^{-}(q),
$$

for all $q \in Q$ and $j=1,2, \cdots, m$. This shows that $C^{c}=\left\{\left\langle q,\left(\left[1-\mu_{j}^{+}(q), 1-\mu_{j}^{-}(q)\right], 1-\right.\right.\right.$ $\left.\left.\left.\mu_{j}(q)\right)_{j=1}^{m}\right\rangle: q \in Q\right\}$ is also an ICmPFS.

Remark 1. Since ECMPFS is the negation of ICMPFS, and a certain CmPFS falls in exactly one of the two categories (by definition), the above characterization immediately characterizes the closeness of the set of all ECMPFSs on a certain discourse X. 
Theorem 2. For a collection of ICmPFNs $A_{i}=\left(\left[\mu_{i j}^{-}, \mu_{i j}^{+}\right], \mu_{i j}\right)_{j=1}^{m}, i \in \Omega, P$-maximum and P-minimum are also ICMPFN.

Proof. Since $A_{i}^{\prime} s$ are ICmPFNs, $\mu_{i j}^{-}(x) \leq \mu_{i j}(x) \leq \mu_{i j}^{+}(x)$. This implies that

$$
\sup _{i \in \Omega} \mu_{i j}^{-}(x) \leq \sup _{i \in \Omega} \mu_{i j}(x) \leq \sup _{i \in \Omega} \mu_{i j}^{+}(x)
$$

and

$$
\inf _{i \in \Omega} \mu_{i j}^{-}(x) \leq \inf _{i \in \Omega} \mu_{i j}(x) \leq \inf _{i \in \Omega} \mu_{i j}^{+}(x), j=1,2, \cdots, m .
$$

This shows that $\vee_{P} A_{i}=\left(\left[\sup _{i \in \Omega} \mu_{i j}^{-},_{\sup _{i \in \Omega}} \mu_{i j}^{+}\right], \sup _{i \in \Omega} \mu_{i j}\right)_{j=1}^{m}$ and $\wedge_{P} A_{i}=\left(\left[\inf _{i \in \Omega} \mu_{i j}^{-}\right.\right.$, $\left.\left.\inf _{i \in \Omega} \mu_{i j}^{+}\right], \inf _{i \in \Omega} \mu_{i j}\right)_{j=1}^{m}$ are also ICmPFS.

Remark 2. R-minimum and R-maximum of ICmPFNs may not be ICmPFN. Similarly, $R$ minimum, R-maximum, P-minimum and P-maximum of ECmPFNs may not be ECmPFN. The counter examples are easy to compute.

In any decision-making process, ranking is a basic tool. Decision makers are required to rank the uncertainties on the basis of which the most favorite alternative is filtered. To help decision makers rank the vagueness in CmPF environment, we define score and accuracy functions for CmPFNs.

Definition 11. Let $\check{\mathbb{A}}=\left(\Im_{j}, \wp_{j}\right)_{j=1}^{m}$ be a CmPFN. The score and accuracy functions are, respectively, defined as

$$
S(\check{\mathbb{A}})=\frac{\sum_{j=1}^{m}\left|\ell\left(\Im_{j}\right)-\wp_{j}\right|}{m}
$$

and

$$
\alpha(\check{\mathbb{A}})=\frac{\sum_{j=1}^{m}\left(\ell\left(\Im_{j}\right)+\wp_{j}\right)}{2 m},
$$

where $\ell\left(\Im_{j}\right)$ is the length of the fuzzy interval $\Im_{j}$. It is clear that $S(\check{\mathbb{A}}) \in[-1,1]$ and $\alpha(\check{\mathbb{A}}) \in[0,1]$.

Proposition 1. The ranking of CMPFNs with the help of the proposed score and accuracy functions is observed as follows.

If $\mathbb{A}_{\mathfrak{m}}$ and $\mathbb{B}_{\mathfrak{m}}$ are two CmPFNs. Then

- $\quad \mathbb{A}_{\mathfrak{m}}<\mathbb{B}_{\mathfrak{m}}$ if $S\left(\mathbb{A}_{\mathfrak{m}}\right)<S\left(\mathbb{B}_{\mathfrak{m}}\right)$,

- If $S\left(\mathbb{A}_{\mathfrak{m}}\right)=S\left(\mathbb{B}_{\mathfrak{m}}\right)$, then $\mathbb{A}_{\mathfrak{m}}<\mathbb{B}_{\mathfrak{m}}$ if $\alpha\left(\mathbb{A}_{\mathfrak{m}}\right)<\alpha\left(\mathbb{B}_{\mathfrak{m}}\right)$,

- If, however, $S\left(\mathbb{A}_{\mathfrak{m}}\right)=S\left(\mathbb{B}_{\mathfrak{m}}\right)$ and $\alpha\left(\mathbb{A}_{\mathfrak{m}}\right)=\alpha\left(\mathbb{B}_{\mathfrak{m}}\right)$, then $\mathbb{A}_{\mathfrak{m}}=\mathbb{B}_{\mathfrak{m}}$.

Definition 12. Let $\mathbb{A}_{\mathfrak{m}}=\left\langle\left[\mu_{1}^{-}, \mu_{1}^{+}\right],\left[\mu_{2}^{-}, \mu_{2}^{+}\right], \cdots,\left[\mu_{m}^{-}, \mu_{m}^{+}\right], \mu_{1}, \mu_{2}, \cdots, \mu_{m}\right\rangle=\left\langle\left[\mu_{j}^{-}, \mu_{j}^{+}\right]\right.$, $\left.\mu_{j}\right\rangle_{j=1}^{m}$ and $\mathbb{B}_{\mathfrak{m}}=\left\langle\left[v_{1}^{-}, v_{1}^{+}\right],\left[v_{2}^{-}, v_{2}^{+}\right], \cdots,\left[v_{m}^{-}, v_{m}^{+}\right], v_{1}, v_{2}, \cdots, v_{m}\right\rangle=\left\langle\left[v_{j}^{-}, v_{j}^{+}\right], v_{j}\right\rangle_{j=1}^{m}$ be two cubic m-polar fuzzy sets.

The distance between the two CmPFSs is defined by

$$
d\left(\mathbb{A}_{\mathfrak{m}}, \mathbb{B}_{\mathfrak{m}}\right)=\left[\sum_{j=1}^{m}\left|\frac{\mu_{j}^{-}+\mu_{j}^{+}}{2}-\frac{v_{j}^{-}+v_{j}^{+}}{2}\right|^{m}+\sum_{j=1}^{m}\left|\mu_{j}-v_{j}\right|^{m}\right]^{1 / m} .
$$




\section{Extension of Dombi's T-norm and T-conorm to CmPFSs}

In 1982, Dombi [26] proposed some special kinds of t-conorm and t-norm. These notions laid the foundation of various operations in different uncertainty environments. On the basis of these operations, various kinds of aggregation operators (AOs) were defined, which made the MCDM process very effective. Dombi t-conorm and t-norm are, respectively, defined as follows:

$$
\begin{gathered}
\operatorname{Dom}^{*}(k, p)=1-\frac{1}{1+\left\{\left(\frac{k}{1-k}\right)^{s}+\left(\frac{p}{1-p}\right)^{s}\right\}^{1 / s}} \\
\operatorname{Dom}(k, p)=\frac{1}{1+\left\{\left(\frac{1-k}{k}\right)^{s}+\left(\frac{1-p}{p}\right)^{s}\right\}^{1 / s}}
\end{gathered}
$$

where $s \geq 1$ and $k, p \in[0,1]$.

\subsection{Dombi P-operations for Cubic M-polar Fuzzy Environment}

Owing to Dombi t-conorm and t-norm, we define some basic Dombi P-operations for CmPFS. Let $\mathbb{A}_{\mathfrak{m}}=\left\{\left(x,\left[\mu_{1}^{-}(x), \mu_{1}^{+}(x)\right],\left[\mu_{2}^{-}(x), \mu_{2}^{+}(x)\right], \cdots,\left[\mu_{m}^{-}(x), \mu_{m}^{+}(x)\right], \mu_{1}(x), \mu_{2}(x), \cdots\right.\right.$, $\left.\left.\mu_{m}(x)\right): x \in X\right\}$ and $\mathbb{B}_{\mathfrak{m}}=\left\{\left(x,\left[v_{1}^{-}(x), v_{1}^{+}(x)\right],\left[v_{2}^{-}(x), v_{2}^{+}(x)\right], \cdots,\left[v_{m}^{-}(x), v_{m}^{+}(x)\right], v_{1}(x)\right.\right.$, $\left.\left.v_{2}(x), \cdots, v_{m}(x)\right): x \in X\right\}$ be two CmPFSs with underlying set $X$. Then

- $\quad \mathbb{A}_{\mathfrak{m}} \oplus_{P} \mathbb{B}_{\mathfrak{m}}=\left\{\left(x,\left[1-\frac{1}{1+\left\{\left(\frac{\mu_{j}^{-}(x)}{1-\mu_{j}^{-}(x)}\right)^{s}+\left(\frac{v_{j}^{-}(x)}{1-v_{j}^{-}(x)}\right)^{s}\right\}^{1 / s}}, 1-\frac{1}{1+\left\{\left(\frac{\mu_{j}^{+}(x)}{1-\mu_{j}^{+}(x)}\right)^{s}+\left(\frac{v_{j}^{+}(x)}{1-v_{j}^{+}(x)}\right)^{s}\right\}^{1 / s}}\right]\right.\right.$,

$$
\left.\left.1-\frac{1}{1+\left\{\left(\frac{\mu_{j}(x)}{1-\mu_{j}(x)}\right)^{s}+\left(\frac{v_{j}(x)}{1-v_{j}(x)}\right)^{s}\right\}^{1 / s}}\right)_{j=1}^{m}\right\}
$$

- $\quad \mathbb{A}_{\mathfrak{m}} \otimes_{P} \mathbb{B}_{\mathfrak{m}}=\left\{\left(x,\left[\frac{1}{1+\left\{\left(\frac{1-\mu_{j}^{-}(x)}{\mu_{j}^{-}(x)}\right)^{s}+\left(\frac{1-v_{j}^{-}(x)}{v_{j}^{-}(x)}\right)^{s}\right\}^{1 / s}}, \frac{1}{1+\left\{\left(\frac{1-\mu_{j}^{+}(x)}{\mu_{j}^{+}(x)}\right)^{s}+\left(\frac{1-v_{j}^{+}(x)}{v_{j}^{+}(x)}\right)^{s}\right\}^{1 / s}}\right]\right.\right.$,

$$
\left.\left.\frac{1}{1+\left\{\left(\frac{1-\mu_{j}(x)}{\mu_{j}(x)}\right)^{s}+\left(\frac{1-v_{j}(x)}{v_{j}(x)}\right)^{s}\right\}^{1 / s}}\right)_{j=1}^{m}\right\}
$$

- (P-Scalar Multiplication)

$\lambda \mathbb{A}_{\mathfrak{m}}=\left\{\left(x,\left[1-\frac{1}{1+\left\{\lambda\left(\frac{\mu_{j}^{-}(x)}{1-\mu_{j}^{-}(x)}\right)^{s}\right\}^{1 / s}}, 1-\frac{1}{1+\left\{\lambda\left(\frac{\mu_{j}^{+}(x)}{1-\mu_{j}^{+}(x)}\right)^{s}\right\}^{1 / s}}\right], 1-\frac{1}{1+\left\{\lambda\left(\frac{\mu_{j}(x)}{1-\mu_{j}(x)}\right)^{s}\right\}^{1 / s}}\right)_{j=1}^{m}\right\}$

- (P-Power)

$\mathbb{A}_{\mathfrak{m}}^{\lambda}=\left\{\left(x,\left[\frac{1}{1+\left\{\lambda\left(\frac{1-\mu_{j}^{-}(x)}{\mu_{j}^{-}(x)}\right)^{s}\right\}^{1 / s}}, \frac{1}{1+\left\{\lambda\left(\frac{1-\mu_{j}^{+}(x)}{\mu_{j}^{+}(x)}\right)^{s}\right\}^{1 / s}}\right], \frac{1}{1+\left\{\lambda\left(\frac{1-\mu_{j}(x)}{\mu_{j}(x)}\right)^{s}\right\}^{1 / s}}\right)_{j=1}^{m}\right\}$

where $s \geqslant 1$.

Theorem 3. Let $\mathbb{A}_{\mathfrak{m}}, \mathbb{B}_{\mathfrak{m}}$ and $\mathbb{C}_{\mathfrak{m}}$ be the CmPFSs. Then

1. $\quad \mathbb{A}_{\mathfrak{m}} \oplus_{P} \mathbb{B}_{\mathfrak{m}}=\mathbb{B}_{\mathfrak{m}} \oplus_{P} \mathbb{A}_{\mathfrak{m}}$

2. $\quad \mathbb{A}_{\mathfrak{m}} \otimes_{P} \mathbb{B}_{\mathfrak{m}}=\mathbb{B}_{\mathfrak{m}} \otimes_{P} \mathbb{A}_{\mathfrak{m}}$

3. $\quad \mathbb{A}_{\mathfrak{m}} \oplus_{P}\left(\mathbb{B}_{\mathfrak{m}} \oplus_{P} \mathbb{C}_{\mathfrak{m}}\right)=\left(\mathbb{A}_{\mathfrak{m}} \oplus_{P} \mathbb{B}_{\mathfrak{m}}\right) \oplus_{P} \mathbb{C}_{\mathfrak{m}}=\mathbb{A}_{\mathfrak{m}} \oplus_{P} \mathbb{B}_{\mathfrak{m}} \oplus_{P} \mathbb{C}_{\mathfrak{m}}$

4. $\quad \mathbb{A}_{\mathfrak{m}} \otimes_{P}\left(\mathbb{B}_{\mathfrak{m}} \otimes_{P} \mathbb{C}_{\mathfrak{m}}\right)=\left(\mathbb{A}_{\mathfrak{m}} \otimes_{P} \mathbb{B}_{\mathfrak{m}}\right) \otimes_{P} \mathbb{C}_{\mathfrak{m}}=\mathbb{A}_{\mathfrak{m}} \otimes_{P} \mathbb{B}_{\mathfrak{m}} \otimes_{P} \mathbb{C}_{\mathfrak{m}}$

5. $\mu\left(\lambda \mathbb{A}_{\mathfrak{m}}\right)=(\mu \lambda) \mathbb{A}_{\mathfrak{m}}$

6. $\lambda\left(\mathbb{A}_{\mathfrak{m}} \oplus_{P} \mathbb{B}_{\mathfrak{m}}\right)=\lambda \mathbb{A}_{\mathfrak{m}} \oplus_{P} \lambda \mathbb{B}_{\mathfrak{m}}$

7. $\quad \lambda\left(\mathbb{A}_{\mathfrak{m}} \otimes_{P} \mathbb{B}_{\mathfrak{m}}\right)=\lambda \mathbb{A}_{\mathfrak{m}} \otimes_{P} \lambda \mathbb{B}_{\mathfrak{m}}$ 

8. $\quad\left(\mathbb{A}_{\mathfrak{m}} \oplus_{P} \mathbb{B}_{\mathfrak{m}}\right)^{\lambda}=\mathbb{A}_{\mathfrak{m}}^{\lambda} \oplus_{P} \mathbb{B}_{\mathfrak{m}}^{\lambda}$
9. $\quad\left(\mathbb{A}_{\mathfrak{m}} \otimes_{P} \mathbb{B}_{\mathfrak{m}}\right)^{\lambda}=\mathbb{A}_{\mathfrak{m}}^{\lambda} \otimes_{P} \mathbb{B}_{\mathfrak{m}}^{\lambda}$
10. $\left(\mathbb{A}_{\mathfrak{m}}^{\lambda}\right)^{\mu}=\mathbb{A}_{\mathfrak{m}}^{\lambda \mu}=\mathbb{A}_{\mathfrak{m}}^{\mu \lambda}$

Proof. We prove (without any loss) our claim by considering CmPFNs, $\mathbb{A}_{\mathfrak{m}}=\left(\left[\mu_{j}^{-}, \mu_{j}^{+}\right]\right.$, $\left.\mu_{j}\right)_{j=1}^{m}, \mathbb{B}_{\mathfrak{m}}=\left(\left[v_{j}^{-}, v_{j}^{+}\right], v_{j}\right)_{j=1}^{m}$ and $\mathbb{C}_{\mathfrak{m}}=\left(\left[\omega_{j}^{-}, \omega_{j}^{+}\right], \omega_{j}\right)_{j=1}^{m}$, corresponding to the alternative $x \in X$. We only prove the statements for lower fuzzy numbers. The rest of the cases are similar.

1. 2. The proof follows from definition.

3. $\left(\mathbb{A}_{\mathfrak{m}} \oplus_{P} \mathbb{B}_{\mathfrak{m}}\right) \oplus_{P} \mathbb{C}_{\mathfrak{m}}=1-\frac{1}{1+\left\{\left(\frac{\mu_{j}^{-}}{1-\mu_{j}^{-}}\right)^{s}+\left(\frac{v_{j}^{-}}{1-v_{j}^{-}}\right)^{s}\right\}^{1 / s}} \oplus_{P} \omega_{j}^{-}$

$$
\begin{aligned}
& =1-\frac{1}{1+\left\{\left(\frac{1-\frac{1}{1+\left\{\left(\frac{\mu_{j}^{-}}{1-\mu_{j}^{-}}\right)^{s}+\left(\frac{v_{j}^{-}}{1-v_{j}^{-}}\right)^{s}\right\}^{1 / s}}}{1+\left\{\left(\frac{\mu_{j}^{-}}{1-\mu_{j}^{-}}\right)^{s}+\left(\frac{v_{j}^{-}}{1-v_{j}^{-}}\right)^{s}\right\}^{1 / s}}\right)^{s}+\left(\frac{\omega_{j}^{-}}{1-\omega_{j}^{-}}\right)^{s}\right\}^{1 / s}} \\
& =1-\frac{1}{1+\left\{\left(\frac{\mu_{j}^{-}}{1-\mu_{j}^{-}}\right)^{s}+\left(\frac{v_{j}^{-}}{1-v_{j}^{-}}\right)^{s}+\left(\frac{\omega_{j}^{-}}{1-\omega_{j}^{-}}\right)^{s}\right\}^{1 / s}} \\
& =1-\frac{1+\left\{\left(\frac{\mu_{j}^{-}}{1-\mu_{j}^{-}}\right)^{s}+\left(\frac{1+\left\{\left(\frac{v_{j}^{-}}{1-v_{j}^{-}}\right)^{s}+\left(\frac{\omega_{j}^{-}}{1-\omega_{j}^{-}}\right)^{s}\right\}^{1 / s}}{1+\left\{\left(\frac{v_{j}^{-}}{1-v_{j}^{-}}\right)^{s}+\left(\frac{\omega_{j}^{-}}{1-\omega_{j}^{-}}\right)^{s}\right\}^{1 / s}}\right\}^{s}\right\}^{1 / s}}{1-\frac{1}{1-\omega^{-}}} \\
& =\mu_{j}^{-} \oplus_{P} 1-\frac{1}{1+\left\{\left(\frac{v_{j}^{-}}{1-v_{j}^{-}}\right)^{s}+\left(\frac{\omega_{j}^{-}}{1-\omega_{j}^{-}}\right)^{s}\right\}^{1 / s}} \\
& =\mathbb{A}_{\mathfrak{m}} \oplus_{P}\left(\mathbb{B}_{\mathfrak{m}} \oplus_{P} \mathbb{C}_{\mathfrak{m}}\right)
\end{aligned}
$$

4. Similar to 3 .

$$
\begin{aligned}
& \text { 5. } \lambda \mathbb{A}_{\mathfrak{m}}=1-\frac{1}{1+\left\{\lambda\left(\frac{\mu_{j}^{-}}{1-\mu_{j}^{-}}\right)^{s}\right\}^{1 / s}} \\
& \Rightarrow \mu\left(\lambda \mathbb{A}_{\mathfrak{m}}\right)=1-\frac{1}{1+\left\{\mu\left(\frac{1+\frac{1}{1+\left\{\lambda\left(\frac{\mu_{j}^{-}}{1-\mu_{j}^{-}}\right)^{s}\right\}^{1 / s}}}{\frac{1}{1+\left\{\lambda\left(\frac{\mu_{j}^{-}}{1-\mu_{j}^{-}}\right)^{s}\right\}^{1 / s}}}\right)^{s}\right\}^{1 / s}} \\
& \Rightarrow \mu\left(\lambda \mathbb{A}_{\mathfrak{m}}\right)=1-\frac{1}{1+\left\{\mu \lambda\left(\frac{\mu_{j}^{-}}{1-\mu_{j}^{-}}\right)^{s}\right\}^{1 / s}}=(\mu \lambda) \mathbb{A}_{\mathfrak{m}} \\
& \text { 6. } \lambda\left(\mathbb{A}_{\mathfrak{m}} \oplus_{P} \mathbb{B}_{\mathfrak{m}}\right)=1-\frac{1}{1+\left\{\lambda\left(\frac{\mu_{j}^{-}}{1-\mu_{j}^{-}}\right)^{s}+\lambda\left(\frac{v_{j}^{-}}{1-v_{j}^{-}}\right)^{s}\right\}^{1 / s}}
\end{aligned}
$$

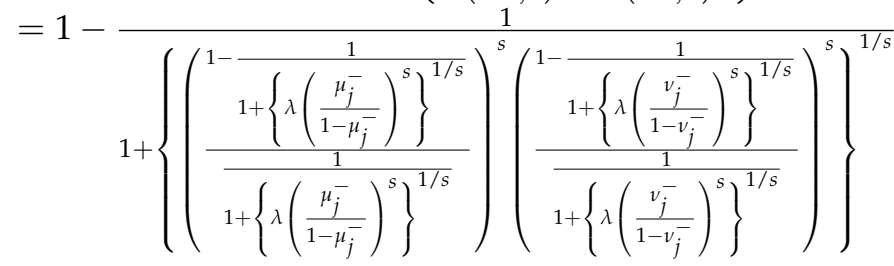


$=1-\frac{1}{1+\left\{\lambda\left(\frac{\mu_{j}^{-}}{1-\mu_{j}^{-}}\right)^{s}\right\}^{1 / s}} \oplus_{P} 1-\frac{1}{1+\left\{\lambda\left(\frac{v_{j}^{-}}{1-v_{j}^{-}}\right)^{s}\right\}^{1 / s}}$

$=\lambda \mathbb{A}_{\mathfrak{m}} \oplus_{P} \lambda \mathbb{B}_{\mathfrak{m}}$

7. 8. 9. Similar to 6 .

10. Follows from definition.

Theorem 4. Let $\mathbb{A}_{\mathfrak{m}}=\left(\left[\mu_{j}^{-}, \mu_{j}^{+}\right], \mu_{j}\right)_{j=1}^{m}$ and $\mathbb{B}_{\mathfrak{m}}=\left(\left[v_{j}^{-}, v_{j}^{+}\right], v_{j}\right)_{j=1}^{m}$ be two ICmPFSs (we are referring to CmPFNs as CmPFSs without any loss). Then $\mathbb{A}_{\mathfrak{m}} \oplus_{P} \mathbb{B}_{\mathfrak{m}}, \mathbb{A}_{\mathfrak{m}} \otimes_{P} \mathbb{B}_{\mathfrak{m}}, \lambda \mathbb{A}_{\mathfrak{m}}$ (P-scalar multiplication) and $\mathbb{A}_{\mathfrak{m}}^{\lambda}$ (P-power) are are also ICmPFS.

Proof. Since $\mathbb{A}_{\mathfrak{m}}$ is ICmPFS, so $\mu_{j}^{-} \leq \mu_{j} \leq \mu_{j}^{+} \Rightarrow 1-\mu_{j}^{+} \leq 1-\mu_{j} \leq 1-\mu_{j}^{-} \Rightarrow \frac{1}{1-\mu_{j}^{-}} \leq$ $\frac{1}{1-\mu_{j}} \leq \frac{1}{1-\mu_{j}^{+}} \Rightarrow \frac{\mu_{j}^{-}}{1-\mu_{j}^{-}} \leq \frac{\mu_{j}}{1-\mu_{j}} \leq \frac{\mu_{j}^{+}}{1-\mu_{j}^{+}} \Rightarrow\left(\frac{\mu_{j}^{-}}{1-\mu_{j}^{-}}\right)^{s} \leq\left(\frac{\mu_{j}}{1-\mu_{j}}\right)^{s} \leq\left(\frac{\mu_{j}^{+}}{1-\mu_{j}^{+}}\right)^{s}$.

Similarly for ICmPFS $\mathbb{B}_{\mathfrak{m}},\left(\frac{v_{j}^{-}}{1-v_{j}^{-}}\right)^{s} \leq\left(\frac{v_{j}}{1-v_{j}}\right)^{s} \leq\left(\frac{v_{j}^{+}}{1-v_{j}^{+}}\right)^{s}$.

Adding both inequalities, we have

$\left(\frac{\mu_{j}^{-}}{1-\mu_{j}^{-}}\right)^{s}+\left(\frac{v_{j}^{-}}{1-v_{j}^{-}}\right)^{s} \leq\left(\frac{\mu_{j}}{1-\mu_{j}}\right)^{s}+\left(\frac{v_{j}}{1-v_{j}}\right)^{s} \leq\left(\frac{\mu_{j}^{+}}{1-\mu_{j}^{+}}\right)^{s}+\left(\frac{v_{j}^{+}}{1-v_{j}^{+}}\right)^{s}$.

$\Rightarrow 1-\frac{1}{1+\left\{\left(\frac{\mu_{j}^{-}}{1-\mu_{j}^{-}}\right)^{s}+\left(\frac{v_{j}^{-}}{1-v_{j}^{-}}\right)^{s}\right\}^{1 / s}} \leq 1-\frac{1}{1+\left\{\left(\frac{\mu_{j}}{1-\mu_{j}}\right)^{s}+\left(\frac{v_{j}}{1-v_{j}}\right)^{s}\right\}^{1 / s}} \leq 1-$

$1+\left\{\left(\frac{\mu_{j}^{+}}{1-\mu_{j}^{+}}\right)^{s}+\left(\frac{v_{j}^{+}}{1-v_{j}^{+}}\right)^{s}\right\}^{1 / s}$,

for all $j=1,2, \cdots, m$.

Following the same root, it can be easily proved that $\mathbb{A}_{\mathfrak{m}} \otimes_{P} \mathbb{B}_{\mathfrak{m}}, \lambda \mathbb{A}_{\mathfrak{m}}$ and $\mathbb{A}_{\mathfrak{m}}^{\lambda}$ are also ICmPFSs.

Remark 3. If $\mathbb{A}_{\mathfrak{m}}$ and $\mathbb{B}_{\mathfrak{m}}$ are ECmPFSs, then $\mathbb{A}_{\mathfrak{m}} \oplus_{P} \mathbb{B}_{\mathfrak{m}}, \mathbb{A}_{\mathfrak{m}} \otimes_{P} \mathbb{B}_{\mathfrak{m}}, \lambda \mathbb{A}_{\mathfrak{m}}$, and $\mathbb{A}_{\mathfrak{m}}^{\lambda}$ may not be ECmPFSs. Counter examples are easy to compute.

\subsection{Dombi R-operations for Cubic M-polar Fuzzy Sets}

Let $\mathbb{A}_{\mathfrak{m}}$ and $\mathbb{B}_{\mathfrak{m}}$ be the CmPFSs as mentioned in Section 2.1. Then

- $\quad \mathbb{A}_{\mathfrak{m}} \oplus_{R} \mathbb{B}_{\mathfrak{m}}=\left\{\left(x,\left[1-\frac{1}{1+\left\{\left(\frac{\mu_{j}^{-}(x)}{1-\mu_{j}^{-}(x)}\right)^{s}+\left(\frac{v_{j}^{-}(x)}{1-v_{j}^{-}(x)}\right)^{s}\right\}^{1 / s}}, 1-\frac{1}{1+\left\{\left(\frac{\mu_{j}^{+}(x)}{1-\mu_{j}^{+}(x)}\right)^{s}+\left(\frac{v_{j}^{+}(x)}{1-v_{j}^{+}(x)}\right)^{s}\right\}^{1 / s}}\right]\right.\right.$,

$\left.\left.\frac{1}{1+\left\{\left(\frac{1-\mu_{j}(x)}{\mu_{j}(x)}\right)^{s}+\left(\frac{1-v_{j}(x)}{v_{j}(x)}\right)^{s}\right\}^{1 / s}}\right)_{j=1}^{m}\right\}$

- $\quad \mathbb{A}_{\mathfrak{m}} \otimes_{R} \mathbb{B}_{\mathfrak{m}}=\left\{\left(x,\left[\frac{1}{1+\left\{\left(\frac{1-\mu_{j}^{-}(x)}{\mu_{j}^{-}(x)}\right)^{s}+\left(\frac{1-v_{j}^{-}(x)}{v_{j}^{-}(x)}\right)^{s}\right\}^{1 / s}}, \frac{1}{1+\left\{\left(\frac{1-\mu_{j}^{+}(x)}{\mu_{j}^{+}(x)}\right)^{s}+\left(\frac{1-v_{j}^{+}(x)}{v_{j}^{+}(x)}\right)^{s}\right\}^{1 / s}}\right]\right.\right.$,

$$
\left.\left.1-\frac{1}{1+\left\{\left(\frac{\mu_{j}(x)}{1-\mu_{j}(x)}\right)^{s}+\left(\frac{v_{j}(x)}{1-v_{j}(x)}\right)^{s}\right\}^{1 / s}}\right)_{j=1}^{m}\right\}
$$

- (R-Scalar Multiplication)

$$
\lambda \mathbb{A}_{\mathfrak{m}}=\left\{\left(x,\left[1-\frac{1}{1+\left\{\lambda\left(\frac{\mu_{j}^{-}(x)}{1-\mu_{j}^{-}(x)}\right)^{s}\right\}^{1 / s}}, 1-\frac{1}{1+\left\{\lambda\left(\frac{\mu_{j}^{+}(x)}{1-\mu_{j}^{+}(x)}\right)^{s}\right\}^{1 / s}}\right], \frac{1}{1+\left\{\lambda\left(\frac{1-\mu_{j}(x)}{\mu_{j}(x)}\right)^{s}\right\}^{1 / s}}\right)_{j=1}^{m}\right\}
$$


- $\quad($ R-Power)

$$
\mathbb{A}_{\mathfrak{m}}^{\lambda}=\left\{\left(x,\left[\frac{1}{1+\left\{\lambda\left(\frac{1-\mu_{j}^{-}(x)}{\mu_{j}^{-}(x)}\right)^{s}\right\}^{1 / s}}, \frac{1}{1+\left\{\lambda\left(\frac{1-\mu_{j}^{+}(x)}{\mu_{j}^{+}(x)}\right)^{s}\right\}^{1 / s}}\right], 1-\frac{1}{1+\left\{\lambda\left(\frac{\mu_{j}(x)}{1-\mu_{j}(x)}\right)^{s}\right\}^{1 / s}}\right)_{j=1}^{m}\right\}
$$

where $s \geqslant 1$.

Theorem 5. Let $\mathbb{A}_{\mathfrak{m}}, \mathbb{B}_{\mathfrak{m}}$ and $\mathbb{C}_{\mathfrak{m}}$ be the CmPFSs. Then

1. $\mathbb{A}_{\mathfrak{m}} \oplus_{R} \mathbb{B}_{\mathfrak{m}}=\mathbb{B}_{\mathfrak{m}} \oplus_{R} \mathbb{A}_{\mathfrak{m}}$

2. $\quad \mathbb{A}_{\mathfrak{m}} \otimes_{R} \mathbb{B}_{\mathfrak{m}}=\mathbb{B}_{\mathfrak{m}} \otimes_{R} \mathbb{A}_{\mathfrak{m}}$

3. $\mathbb{A}_{\mathfrak{m}} \oplus_{R}\left(\mathbb{B}_{\mathfrak{m}} \oplus_{R} \mathbb{C}_{\mathfrak{m}}\right)=\left(\mathbb{A}_{\mathfrak{m}} \oplus_{R} \mathbb{B}_{\mathfrak{m}}\right) \oplus_{R} \mathbb{C}_{\mathfrak{m}}=\mathbb{A}_{\mathfrak{m}} \oplus_{R} \mathbb{B}_{\mathfrak{m}} \oplus_{R} \mathbb{C}_{\mathfrak{m}}$

4. $\quad \mathbb{A}_{\mathfrak{m}} \otimes_{R}\left(\mathbb{B}_{\mathfrak{m}} \otimes_{R} \mathbb{C}_{\mathfrak{m}}\right)=\left(\mathbb{A}_{\mathfrak{m}} \otimes_{R} \mathbb{B}_{\mathfrak{m}}\right) \otimes_{R} \mathbb{C}_{\mathfrak{m}}=\mathbb{A}_{\mathfrak{m}} \otimes_{R} \mathbb{B}_{\mathfrak{m}} \otimes_{R} \mathbb{C}_{\mathfrak{m}}$

5. $\quad \mu\left(\lambda \mathbb{A}_{\mathfrak{m}}\right)=(\mu \lambda) \mathbb{A}_{\mathfrak{m}}$

6. $\quad \lambda\left(\mathbb{A}_{\mathfrak{m}} \oplus_{R} \mathbb{B}_{\mathfrak{m}}\right)=\lambda \mathbb{A}_{\mathfrak{m}} \oplus_{R} \lambda \mathbb{B}_{\mathfrak{m}}$

7. $\quad \lambda\left(\mathbb{A}_{\mathfrak{m}} \otimes_{R} \mathbb{B}_{\mathfrak{m}}\right)=\lambda \mathbb{A}_{\mathfrak{m}} \otimes_{R} \lambda \mathbb{B}_{\mathfrak{m}}$

8. $\quad\left(\mathbb{A}_{\mathfrak{m}} \oplus_{R} \mathbb{B}_{\mathfrak{m}}\right)^{\lambda}=\mathbb{A}_{\mathfrak{m}}^{\lambda} \oplus_{R} \mathbb{B}_{\mathfrak{m}}^{\lambda}$

9. $\quad\left(\mathbb{A}_{\mathfrak{m}} \otimes_{R} \mathbb{B}_{\mathfrak{m}}\right)^{\lambda}=\mathbb{A}_{\mathfrak{m}}^{\lambda} \otimes_{R} \mathbb{B}_{\mathfrak{m}}^{\lambda}$

10. $\left(\mathbb{A}_{\mathfrak{m}}^{\lambda}\right)^{\mu}=\mathbb{A}_{\mathfrak{m}}^{\lambda \mu}=\mathbb{A}_{\mathfrak{m}}^{\mu \lambda}$

Proof. Similar to Theorem 3.

Remark 4. If $\mathbb{A}_{\mathfrak{m}}$ and $\mathbb{B}_{\mathfrak{m}}$ are ICmPFNs (or ECmPFNs), then $\mathbb{A}_{\mathfrak{m}} \oplus_{R} \mathbb{B}_{\mathfrak{m}}, \mathbb{A}_{\mathfrak{m}} \otimes_{R} \mathbb{B}_{\mathfrak{m}}, \lambda \mathbb{A}_{\mathfrak{m}}$ (R-Scalar Multiplication), and $\mathbb{A}_{\mathfrak{m}}^{\lambda}$ (R-Power) may not be ICmPFNs (or ECmPFNs). Counter examples can be easily computed.

\section{CmPF Dombi Aggregation Operators with P-order}

In this section, we develop Dombi P-aggregation operators in cubic m-polar fuzzy environment and give a brief description with the help of examples. These are cubic m-polar fuzzy Dombi P-averaging operator (CmPFDPAO), Cubic m-polar fuzzy Dombi weighted P-averaging operator (CMPFDWPAO), and cubic m-polar fuzzy Dombi ordered weighted P-averaging operator (CMPFDOWPAO). We will examine some properties of the proposed aggregation operators as well.

Definition 13. For the family of CmPFNs $\mathbb{A}_{\mathfrak{m}_{1}}, \mathbb{A}_{\mathfrak{m}_{2}}, \cdots, \mathbb{A}_{\mathfrak{m}_{n}}$, the cubic m-polar fuzzy Dombi P-averaging operator is defined as

$$
\operatorname{CmPFDPAO}\left(\mathbb{A}_{\mathfrak{m}_{1}}, \mathbb{A}_{\mathfrak{m}_{2}}, \cdots, \mathbb{A}_{\mathfrak{m}_{n}}\right)=\mathbb{A}_{\mathfrak{m}_{1}} \oplus_{P} \mathbb{A}_{\mathfrak{m}_{2}} \oplus_{P} \cdots \oplus_{P} \mathbb{A}_{\mathfrak{m}_{n}} .
$$

Theorem 6. Let $\mathbb{A}_{\mathfrak{m}_{i}}=\left(\left[\mu_{i j}^{-}, \mu_{i j}^{+}\right], \mu_{i j}\right)_{j=1}^{m}, i=1,2, \cdots, n$, be the family of CmPFNs. Then their aggregated value is again a CmPFN and

$$
\begin{gathered}
\operatorname{CmPFDPAO}\left(\mathbb{A}_{\mathfrak{m}_{1}}, \mathbb{A}_{\mathfrak{m}_{2}, \cdots,} \mathbb{A}_{\mathfrak{m}_{n}}\right)= \\
\left(\left[1-\frac{1}{1+\left\{\sum_{i=1}^{n}\left(\frac{\mu_{i j}^{-}}{1-\mu_{i j}^{-}}\right)^{s}\right\}^{1 / s}}, 1-\frac{1}{1+\left\{\sum_{i=1}^{n}\left(\frac{\mu_{i j}^{+}}{1-\mu_{i j}^{+}}\right)^{s}\right\}^{1 / s}}\right], 1-\frac{1}{1+\left\{\sum_{i=1}^{n}\left(\frac{\mu_{i j}}{1-\mu_{i j}}\right)^{s}\right\}^{1 / s}}\right)_{j=1}^{m}
\end{gathered}
$$

Proof. We can prove it by induction on $n$.

For $n=2$, we have

$$
\operatorname{CmPFDPAO}\left(\mathbb{A}_{\mathfrak{m}_{1}}, \mathbb{A}_{\mathfrak{m}_{2}}\right)=
$$




$$
\left(\left[1-\frac{1}{1+\left\{\left(\frac{\mu_{1 j}^{-}}{1-\mu_{1 j}^{-}}\right)^{s}+\left(\frac{\mu_{2 j}^{-}}{1-\mu_{2 j}^{-}}\right)^{s}\right\}^{1 / s}}, 1-\frac{1}{1+\left\{\left(\frac{\mu_{1 j}^{+}}{1-\mu_{1 j}^{+}}\right)^{s}+\left(\frac{\mu_{2 j}^{+}}{1-\mu_{2 j}^{+}}\right)^{s}\right\}^{1 / s}}\right], 1-\frac{1}{1+\left\{\left(\frac{\mu_{1 j}}{1-\mu_{1 j}}\right)^{s}+\left(\frac{\mu_{2 j}}{1-\mu_{2 j}}\right)^{s}\right\}^{1 / s}}\right)_{j=1}^{m}
$$

which is a CmPFN, by definition.

Suppose $n>2$, and our proposed averaging formula is true for CmPFNs numbered less than $n$.

Now we see that

$\operatorname{CmPFDPAO}\left(\mathbb{A}_{\mathfrak{m}_{1}}, \mathbb{A}_{\mathfrak{m}_{2}}, \cdots, \mathbb{A}_{\mathfrak{m}_{n}}\right)=$

$\left(\left[1-\frac{1}{1+\left\{\sum_{i=1}^{n}\left(\frac{\mu_{i j}^{-}}{1-\mu_{i j}^{-}}\right)^{s}\right\}^{1 / s}}, 1-\frac{1}{1+\left\{\Sigma_{i=1}^{n}\left(\frac{\mu_{i j}^{+}}{1-\mu_{i j}^{+}}\right)^{s}\right\}^{1 / s}}\right], 1-\frac{1}{1+\left\{\Sigma_{i=1}^{n}\left(\frac{\mu_{i j}}{1-\mu_{i j}}\right)^{s}\right\}^{1 / s}}\right)_{j=1}^{m}$

$=\left(\left[1-\frac{1}{1+\left\{\sum_{i=1}^{n-1}\left(\frac{\mu_{i j}^{-}}{1-\mu_{i j}^{-}}\right)^{s}\right\}^{1 / s}}, 1-\frac{1}{1+\left\{\sum_{i=1}^{n-1}\left(\frac{\mu_{i j}^{+}}{1-\mu_{i j}^{+}}\right)^{s}\right\}^{1 / s}}\right], 1-\frac{1}{1+\left\{\sum_{i=1}^{n-1}\left(\frac{\mu_{i j}}{1-\mu_{i j}}\right)^{s}\right\}^{1 / s}}\right)_{j=1}^{m}$

$\oplus_{P}\left(\left[\mu_{n j}^{-}, \mu_{n j}^{+}\right], \mu_{n j}\right)$

which is a CmPFN by induction hypothesis.

Remark 5. Theorem 4 implies that the aggregation of ICmPFNs $\mathbb{A}_{\mathfrak{m}_{1}}, \mathbb{A}_{\mathfrak{m}_{2}}, \cdots, \mathbb{A}_{\mathfrak{m}_{n}}$, under CMPFDPAO is again an ICmPFN. However, there is no assurance about external aggregation.

Example 1. Let us consider four C3PFNs

$\mathbb{A}_{\mathfrak{m}_{1}}=([0.20,0.27],[0.30,0.41],[0.25,0.31], 0.25,0.80,0.25)$

$\mathbb{A}_{\mathfrak{m}_{2}}=([0.21,0.29],[0.29,0.40],[0.21,0.33], 0.28,0.77,0.27)$

$\mathbb{A}_{\mathfrak{m}_{3}}=([0.19,0.25],[0.32,0.38],[0.23,0.29], 0.26,0.82,0.26)$

$\mathbb{A}_{\mathfrak{m}_{4}}=([0.22,0.26],[0.28,0.39],[0.24,0.32], 0.29,0.81,0.28)$.

For $s=4$, the aggregation under CЗPFDPAO is given by

$$
\begin{aligned}
& C m P F D P A O\left(\mathbb{A}_{\mathfrak{m}_{1}}, \mathbb{A}_{\mathfrak{m}_{2}}, \mathbb{A}_{\left.\mathfrak{m}_{3}, \mathbb{A}_{\mathfrak{m}_{4}}\right)=}^{(}\left[1-\frac{1}{1+\left\{\Sigma_{i=1}^{4}\left(\frac{\mu_{i j}^{-}}{1-\mu_{i j}^{-}}\right)^{s}\right\}^{1 / s}}, 1-\frac{1}{1+\left\{\Sigma_{i=1}^{4}\left(\frac{\mu_{i j}^{+}}{1-\mu_{i j}^{+}}\right)^{s}\right\}^{1 / s}}\right], 1-\frac{1}{1+\left\{\Sigma_{i=1}^{4}\left(\frac{\mu_{i j}}{1-\mu_{i j}}\right)^{s}\right\}^{1 / s}}\right)_{j=1}^{3} \\
=( & {\left[1-\frac{1}{1+\left\{\left(\frac{0.2}{1-0.2}\right)^{4}+\left(\frac{0.21}{1-0.21}\right)^{4}+\left(\frac{0.19}{1-0.19}\right)^{4}+\left(\frac{0.22}{1-0.22}\right)^{4}\right\}^{1 / 4}}, 1-\frac{1}{1+\left\{\left(\frac{0.27}{1-0.27}\right)^{4}+\left(\frac{0.29}{1-0.29}\right)^{4}+\left(\frac{0.25}{1-0.25}\right)^{4}+\left(\frac{0.26}{1-0.26}\right)^{4}\right\}^{1 / 4}}\right], } \\
& {\left[1-\frac{1}{1+\left\{\left(\frac{0.30}{1-0.30}\right)^{4}+\left(\frac{0.29}{1-0.29}\right)^{4}+\left(\frac{0.32}{1-0.32}\right)^{4}+\left(\frac{0.28}{1-0.28}\right)^{4}\right\}^{1 / 4}}, 1-\frac{1}{1+\left\{\left(\frac{0.41}{1-0.41}\right)^{4}+\left(\frac{0.40}{1-0.40}\right)^{4}+\left(\frac{0.38}{1-0.38}\right)^{4}+\left(\frac{0.39}{1-0.39}\right)^{4}\right\}^{1 / 4}}\right], } \\
& {\left[1-\frac{1}{1+\left\{\left(\frac{0.25}{1-0.25}\right)^{4}+\left(\frac{0.21}{1-0.21}\right)^{4}+\left(\frac{0.23}{1-0.23}\right)^{4}+\left(\frac{0.24}{1-0.24}\right)^{4}\right\}^{1 / 4}}, 1-\frac{1}{1+\left\{\left(\frac{0.31}{1-0.31}\right)^{4}+\left(\frac{0.33}{1-0.33}\right)^{4}+\left(\frac{0.29}{1-0.29}\right)^{4}+\left(\frac{0.32}{1-0.32}\right)^{4}\right\}^{1 / 4}}\right], } \\
& 1-\frac{1}{1+\left\{\left(\frac{0.25}{1-0.25}\right)^{4}+\left(\frac{0.28}{1-0.28}\right)^{4}+\left(\frac{0.26}{1-0.26}\right)^{4}+\left(\frac{0.29}{1-0.29}\right)^{4}\right\}^{1 / 4}}, 1-\frac{1}{1+\left\{\left(\frac{0.80}{1-0.80}\right)^{4}+\left(\frac{0.77}{1-0.77}\right)^{4}+\left(\frac{0.82}{1-0.82}\right)^{4}+\left(\frac{0.81}{1-0.81}\right)^{4}\right\}^{1 / 4}} \\
& \left.1-\frac{1}{1+\left\{\left(\frac{0.25}{1-0.25}\right)^{4}+\left(\frac{0.27}{1-0.27}\right)^{4}+\left(\frac{0.26}{1-0.26}\right)^{4}+\left(\frac{0.28}{1-0.28}\right)^{4}\right\}^{1 / 4}}\right) \\
= & ([0.27,0.34],[0.35,0.48],[0.30,0.39], 0.35,0.85,0.34) .
\end{aligned}
$$

In the following, we see that CmPFDPAO is commutative.

Theorem 7 (Commutative). Let $\mathbb{A}_{\mathfrak{m}_{i}}=\left(\left[\mu_{i j}^{-}, \mu_{i j}^{+}\right], \mu_{i j}\right)_{j=1}^{m}, i=1,2, \cdots, n$, be the assembly of CmPFNs. Then

$$
\operatorname{CmPFDPAO}\left(\mathbb{A}_{\mathfrak{m}_{1}}, \mathbb{A}_{\mathfrak{m}_{2}}, \cdots, \mathbb{A}_{\mathfrak{m}_{n}}\right)=\operatorname{CmPFDPAO}\left(\mathbb{A}_{\mathfrak{m}_{1}}^{\prime}, \mathbb{A}_{\mathfrak{m}_{2}}^{\prime}, \cdots, \mathbb{A}_{\mathfrak{m}_{n}}^{\prime}\right),
$$

where $\left(\mathbb{A}_{\mathfrak{m}_{i}}\right)_{i=1}^{n}$ is a permutation of $\left(\mathbb{A}_{\mathfrak{m}_{i}}\right)_{i=1}^{n}$. 
Definition 14. For a collection of CmPFNs $A_{1}, A_{2}, \cdots, A_{n}$, the cubic m-polar fuzzy Dombi weighted P-averaging operator is defined as

$$
\operatorname{CmPFDWPAO}\left(A_{1}, A_{2}, \cdots, A_{n}\right)=w_{1} A_{1} \oplus_{P} w_{2} A_{2} \oplus_{P} \cdots \oplus_{P} w_{n} A_{n},
$$

where $w=\left(w_{1}, w_{2}, \cdots, w_{n}\right)$ is a weight vector with $\sum_{j=1}^{n} w_{j}=1$ and $w_{j}>0$.

Theorem 8. Let $A_{i}=\left(\left[\mu_{i j}^{-}, \mu_{i j}^{+}\right], \mu_{i j}\right)_{j=1}^{m}, i=1,2, \cdots, n$, be the collection of CmPFNs. Then their aggregated value under CmPFDWPAO is again a CmPFN and $\operatorname{CmPFDWPAO}\left(A_{1}, A_{2}, \cdots, A_{n}\right)$

$$
=\left(\left[1-\frac{1}{1+\left\{\Sigma_{i=1}^{n} w_{i}\left(\frac{\mu_{i j}^{-}}{1-\mu_{i j}^{-}}\right)^{k}\right\}^{1 / k}}, 1-\frac{1}{1+\left\{\sum_{i=1}^{n} w_{i}\left(\frac{\mu_{i j}^{+}}{1-\mu_{i j}^{+}}\right)^{k}\right\}^{1 / k}}\right], 1-\frac{1}{1+\left\{\Sigma_{i=1}^{n} w_{i}\left(\frac{\mu_{i j}}{1-\mu_{i j}}\right)^{k}\right\}^{1 / k}}\right)_{j=1}^{m}
$$

Proof. We can prove it by induction on $\mathrm{n}$.

For $n=2$, we have

$\operatorname{CmPFDWPAO}\left(A_{1}, A_{2}\right)$

$=\left(\left[1-\frac{1}{1+\left\{w_{1}\left(\frac{\mu_{1 j}^{-}}{1-\mu_{1 j}^{-}}\right)^{k}+w_{2}\left(\frac{\mu_{2 j}^{-}}{1-\mu_{2 j}^{-}}\right)^{k}\right\}^{1 / k}}, 1-\frac{1}{1+\left\{w_{1}\left(\frac{\mu_{1 j}^{+}}{1-\mu_{1 j}^{+}}\right)^{k}+w_{2}\left(\frac{\mu_{2 j}^{+}}{1-\mu_{2 j}^{+}}\right)^{k}\right\}^{1 / k}}\right]\right.$,

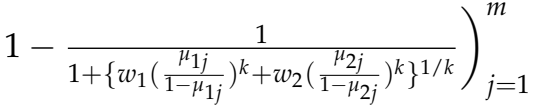

which is a CmPFN, by definition.

Suppose $n>2$, and our proposed averaging formula is true for CmPFNs numbered less than $n$.

Now we see that

$\operatorname{CmPFDWPAO}\left(A_{1}, A_{2}, \cdots, A_{n}\right)=w_{1} A_{1} \oplus_{P} w_{2} A_{2} \oplus_{P} \cdots \oplus_{P} w_{n} A_{n}$

$=\left(\left[1-\frac{1}{1+\left\{\sum_{i=1}^{n} w_{i}\left(\frac{\mu_{i j}^{-}}{1-\mu_{i j}^{-}}\right)^{k}\right\}^{1 / k}}, 1-\frac{1}{1+\left\{\Sigma_{i=1}^{n} w_{i}\left(\frac{\mu_{i j}^{+}}{1-\mu_{i j}^{+}}\right)^{k}\right\}^{1 / k}}\right], 1-\frac{1}{1+\left\{\sum_{i=1}^{n} w_{i}\left(\frac{\mu_{i j}}{1-\mu_{i j}}\right)^{k}\right\}^{1 / k}}\right)_{j=1}^{m}$

$=\left(\left[1-\frac{1}{1+\left\{\Sigma_{i=1}^{n-1} w_{i}\left(\frac{\mu_{i j}^{-}}{1-\mu_{i j}^{-}}\right)^{k}\right\}^{1 / k}}, 1-\frac{1}{1+\left\{\Sigma_{i=1}^{n-1} w_{i}\left(\frac{\mu_{i j}^{+}}{1-\mu_{i j}^{+}}\right)^{k}\right\}^{1 / k}}\right], 1-\frac{1}{1+\left\{\Sigma_{i=1}^{n-1} w_{i}\left(\frac{\mu_{i j}}{1-\mu_{i j}}\right)^{k}\right\}^{1 / k}}\right)_{j=1}^{m}$

$\oplus_{P} w_{n}\left(\left[\mu_{n j}^{-}, \mu_{n j}^{+}\right], \mu_{n j}\right)$

which is surely a CmPFN by induction hypothesis.

Theorem 9. Let $A_{i}=\left(\left[\mu_{i j}^{-}, \mu_{i j}^{+}\right], \mu_{i j}\right)_{j=1}^{m}, i=1,2, \cdots, n$ be the collection of ICmPFNs with a weight vector $w=\left(w_{1}, w_{1}, \cdots, w_{n}\right)$. Then CmPFDWPAO $\left(A_{1}, A_{2}, \cdots, A_{n}\right)$ is also an ICmPFN.

Proof. Since $A_{i}^{\prime} s$ are ICmPFNs, so

$\mu_{i j}^{-} \leq \mu_{i j} \leq \mu_{i j}^{+} \Rightarrow 1-\mu_{i j}^{+} \leq 1-\mu_{i j} \leq 1-\mu_{i j}^{-} \Rightarrow \frac{\mu_{i j}^{-}}{1-\mu_{i j}^{-}} \leq \frac{\mu_{i j}}{1-\mu_{i j}} \leq \frac{\mu_{i j}^{+}}{1-\mu_{i j}^{+}}$

$\Rightarrow \sum_{i=1}^{n} w_{i}\left(\frac{\mu_{i j}^{-}}{1-\mu_{i j}^{-}}\right)^{k} \leq \sum_{i=1}^{n} w_{i}\left(\frac{\mu_{i j}}{1-\mu_{i j}}\right)^{k} \leq \sum_{i=1}^{n} w_{i}\left(\frac{\mu_{i j}^{+}}{1-\mu_{i j}^{+}}\right)^{k}$

$\Rightarrow 1-\frac{1}{1+\left\{\sum_{i=1}^{n} w_{i}\left(\frac{\mu_{i j}^{-}}{1-\mu_{i j}^{-}}\right)^{k}\right\}^{1 / k}} \leq 1-\frac{1}{1+\left\{\sum_{i=1}^{n} w_{i}\left(\frac{\mu_{i j}}{1-\mu_{i j}}\right)^{k}\right\}^{1 / k}} \leq 1-\frac{1}{1+\left\{\sum_{i=1}^{n} w_{i}\left(\frac{\mu_{i j}^{+}}{1-\mu_{i j}^{+}}\right)^{k}\right\}^{1 / k}}$,

for all $j=1,2, \cdots, m$. This proves our claim.

Example 2. Consider the data of Example 1 and let the weights assigned to $A_{i}^{\prime} s$ be $(0.31,0.42,0.17$, $0.10)^{t}$. The dictation under CmPFDWPAO $($ fork $=4)$ is given by

$\operatorname{CmPFDWPAO}\left(A_{1}, A_{2}, A_{3}, A_{4}\right)$ 


$$
\begin{aligned}
& =\left(\left[1-\frac{1}{1+\left\{\Sigma_{i=1}^{4} w_{i}\left(\frac{\mu_{i j}^{-}}{1-\mu_{i j}^{-}}\right)^{k}\right\}^{1 / k}}, 1-\frac{1}{1+\left\{\Sigma_{i=1}^{4} w_{i}\left(\frac{\mu_{i j}^{+}}{1-\mu_{i j}^{+}}\right)^{k}\right\}^{1 / k}}\right], 1-\frac{1}{1+\left\{\Sigma_{i=1}^{4} w_{i}\left(\frac{\mu_{i j}}{1-\mu_{i j}}\right)^{k}\right\}^{1 / k}}\right)_{j=1}^{3} \\
& =\left(\left[1-\frac{1}{1+\left\{0.31\left(\frac{0.2}{1-0.2}\right)^{4}+0.42\left(\frac{0.21}{1-0.21}\right)^{4}+0.17\left(\frac{0.19}{1-0.19}\right)^{4}+0.10\left(\frac{0.22}{1-0.22}\right)^{4}\right\}^{1 / 4}},\right.\right. \\
& \left.1-\frac{1}{1+\left\{0.31\left(\frac{0.27}{1-0.27}\right)^{4}+0.42\left(\frac{0.29}{1-0.29}\right)^{4}+0.17\left(\frac{0.25}{1-0.25}\right)^{4}+0.10\left(\frac{0.26}{1-0.26}\right)^{4}\right\}^{1 / 4}}\right] \text {, } \\
& {\left[1-\frac{1}{1+\left\{0.31\left(\frac{0.30}{1-0.30}\right)^{4}+0.42\left(\frac{0.29}{1-0.29}\right)^{4}+0.17\left(\frac{0.32}{1-0.32}\right)^{4}+0.10\left(\frac{0.28}{1-0.28}\right)^{4}\right\}^{1 / 4}}\right. \text {, }} \\
& \left.1-\frac{1}{1+\left\{0.31\left(\frac{0.41}{1-0.41}\right)^{4}+0.42\left(\frac{0.40}{1-0.40}\right)^{4}+0.17\left(\frac{0.38}{1-0.38}\right)^{4}+0.10\left(\frac{0.39}{1-0.39}\right)^{4}\right\}^{1 / 4}}\right] \text {, } \\
& {\left[1-\frac{1}{1+\left\{0.31\left(\frac{0.25}{1-0.25}\right)^{4}+0.42\left(\frac{0.21}{1-0.21}\right)^{4}+0.17\left(\frac{0.23}{1-0.23}\right)^{4}+0.10\left(\frac{0.24}{1-0.24}\right)^{4}\right\}^{1 / 4}},\right.} \\
& \left.1-\frac{1}{1+\left\{0.31\left(\frac{0.31}{1-0.31}\right)^{4}+0.42\left(\frac{0.33}{1-0.33}\right)^{4}+0.17\left(\frac{0.29}{1-0.29}\right)^{4}+0.10\left(\frac{0.32}{1-0.32}\right)^{4}\right\}^{1 / 4}}\right] \text {, } \\
& 1-\frac{1}{1+\left\{0.31\left(\frac{0.25}{1-0.25}\right)^{4}+0.42\left(\frac{0.28}{1-0.28}\right)^{4}+0.17\left(\frac{0.26}{1-0.26}\right)^{4}+0.10\left(\frac{0.29}{1-0.29}\right)^{4}\right\}^{1 / 4}} \text {, } \\
& 1-\frac{1}{1+\left\{0.31\left(\frac{0.80}{1-0.80}\right)^{4}+0.42\left(\frac{0.77}{1-0.77}\right)^{4}+0.17\left(\frac{0.82}{1-0.82}\right)^{4}+0.10\left(\frac{0.81}{1-0.81}\right)^{4}\right\}^{1 / 4}} \text {, } \\
& \left.1-\frac{1}{1+\left\{0.31\left(\frac{0.25}{1-0.25}\right)^{4}+0.42\left(\frac{0.27}{1-0.27}\right)^{4}+0.17\left(\frac{0.26}{1-0.26}\right)^{4}+0.10\left(\frac{0.28}{1-0.28}\right)^{4}\right\}^{1 / 4}}\right) \text {. } \\
& =([0.21,0.28],[0.30,0.40],[0.23,0.32], 0.27,0.80,0.26) \text {. }
\end{aligned}
$$

The following properties can be easily proved for CmPFDWPAO.

Theorem 10 (Idempotency). Let $A_{i}=\left(\left[\mu_{i j}^{-}, \mu_{i j}^{+}\right], \mu_{i j}\right)_{j=1}^{m}, i=1,2, \cdots, n$, be the collection of equal CmPFNs, say $A_{i}=A=\left(\left[\mu_{j}^{-}, \mu_{j}^{+}\right], \mu_{j}\right)_{j=1}^{m}$. Then the aggregated value under CmPFDWPAO is again a CmPFN A. Mathematically, CmPFDWPAO $\left(A_{1}, A_{2}, \cdots, A_{n}\right)=A$.

Proof. $\operatorname{CmPFDWPAO}\left(A_{1}, A_{2}, \cdots, A_{n}\right)=w_{1} A_{1} \oplus_{P} w_{2} A_{2} \oplus_{P} \cdots \oplus_{P} w_{n} A_{n}=w_{1} A \oplus_{P}$ $w_{2} A \oplus_{P} \cdots \oplus_{P} w_{n} A$

$$
\begin{aligned}
& =\left(\left[1-\frac{1}{1+\left\{\sum_{i=1}^{n} w_{i}\left(\frac{\mu_{j}^{-}}{1-\mu_{j}^{-}}\right)^{k}\right\}^{1 / k}}, 1-\frac{1}{1+\left\{\sum_{i=1}^{n} w_{i}\left(\frac{\mu_{j}^{+}}{1-\mu_{j}^{+}}\right)^{k}\right\}^{1 / k}}\right], 1-\frac{1}{1+\left\{\sum_{i=1}^{n} w_{i}\left(\frac{\mu_{j}}{1-\mu_{j}}\right)^{k}\right\}^{1 / k}}\right)_{j=1}^{m} \\
& =\left(\left[1-\frac{1}{1+\left\{\left(\frac{\mu_{j}^{-}}{1-\mu_{j}^{-}}\right)^{k}\right\}^{1 / k}}, 1-\frac{1}{1+\left\{\left(\frac{\mu_{j}^{+}}{1-\mu_{j}^{+}}\right)^{k}\right\}^{1 / k}}\right], 1-\frac{1}{1+\left\{\left(\frac{\mu_{j}}{1-\mu_{j}}\right)^{k}\right\}^{1 / k}}\right)_{j=1}^{m} \\
& =\left(\left[\mu_{j}^{-}, \mu_{j}^{+}\right], \mu_{j}\right)_{j=1}^{m}=A \quad \square
\end{aligned}
$$

Theorem 11 (Monotonicity). Let $A_{i}=\left(\left[\mu_{i j}^{-}, \mu_{i j}^{+}\right], \mu_{i j}\right)_{j=1}^{m}$ and $B_{i}=\left(\left[v_{i j}^{-}, v_{i j}^{+}\right], v_{i j}\right)_{j=1}^{m}, i=$ $1,2, \cdots, n$, be the two collections of CmPFNs such that $A_{i} \leq_{P} B_{i}$ for all $i$. Then

$$
\operatorname{CmPFDWPAO}\left(A_{1}, A_{2}, \cdots, A_{n}\right) \leq_{P} \operatorname{CmPFDWPAO}\left(B_{1}, B_{2}, \cdots, B_{n}\right) .
$$

Proof. By our assumption we have

$\mu_{i j}^{-} \leq v_{i j}^{-}$

$\Rightarrow w_{i}\left(\frac{\mu_{i j}^{-}}{1-\mu_{i j}^{-}}\right)^{k} \leq w_{i}\left(\frac{v_{i j}^{-}}{1-v_{i j}^{-}}\right)^{k}$ 


$$
\begin{aligned}
& \Rightarrow\left\{1+\sum_{i=1}^{n} w_{i}\left(\frac{\mu_{i j}^{-}}{1-\mu_{i j}^{-}}\right)^{k}\right\}^{1 / k} \leq\left\{1+\sum_{i=1}^{n} w_{i}\left(\frac{v_{i j}^{-}}{1-v_{i j}^{-}}\right)^{k}\right\}^{1 / k} \\
& \Rightarrow 1-\frac{1}{\left\{1+\sum_{i=1}^{n} w_{i}\left(\frac{\mu_{i j}^{-}}{1-\mu_{i j}^{-}}\right)^{k}\right\}^{1 / k}} \leq 1-\frac{1}{\left\{1+\sum_{i=1}^{n} w_{i}\left(\frac{v_{i j}^{-}}{1-v_{i j}^{-}}\right)^{k}\right\}^{1 / k}} .
\end{aligned}
$$

Using similar observations for $\mu_{i j}^{+} \leq v_{i j}^{+}$and $\mu_{i j} \leq v_{i j}$, the result follows.

Theorem 12 (Boundedness). Let $A_{i}=\left(\left[\mu_{i j}^{-}, \mu_{i j}^{+}\right], \mu_{i j}\right)_{j=1}^{m}, i=1,2, \cdots, n$, be the collection of CmPFNs. We define $\vee_{P} A_{i}=A^{+}$and $\wedge_{P} A_{i}=A^{-}$. Then

$$
A^{-} \leq \operatorname{CmPFDWPAO}\left(A_{1}, A_{2}, \cdots, A_{n}\right) \leq A^{+}
$$

Proof. The proof is straightforward.

Definition 15. Let $A_{1}, A_{2}, \cdots, A_{n}$ be the family of CmPFNs; the cubic m-polar fuzzy Dombi ordered weighted P-averaging operator is defined as

$$
\operatorname{CmpFDOWPAO}\left(A_{1}, A_{2}, \cdots, A_{n}\right)=w_{1} A_{\sigma(1)} \oplus_{P} w_{2} A_{\sigma(2)} \oplus_{P} \cdots \oplus_{P} w_{n} A_{\sigma(n)},
$$

where $w=\left(w_{1}, w_{2}, \cdots, w_{n}\right)$ is a weight vector with $\sum_{j=1}^{n} w_{j}=1$ and $w_{j}>0$, and $\sigma(i)$ is a permutation of $(i)_{i=1}^{n}$ dictating $A_{\sigma(1)} \geq_{P} A_{\sigma(2)} \geq_{P} \cdots \geq_{P} A_{\sigma(n)}$.

Theorem 13. Let $A_{i}=\left(\left[\mu_{i j}^{-}, \mu_{i j}^{+}\right], \mu_{i j}\right)_{j=1}^{m}, i=1,2, \cdots, n$, be the knot of CmPFNs. Then the accumulated/aggregated value under CmPFDOWPAO is a CmPFN and $\operatorname{CmPFDOWPAO}\left(A_{1}, A_{2}, \cdots, A_{n}\right)$ $=\left(\left[1-\frac{1}{1+\left\{\sum_{i=1}^{n} w_{i}\left(\frac{\mu_{\sigma(i) j}^{-}}{1-\mu_{\sigma(i) j}^{-}}\right)^{k}\right\}^{1 / k}}, 1-\frac{1}{1+\left\{\sum_{i=1}^{n} w_{i}\left(\frac{\mu_{\sigma(i) j}^{+}}{1-\mu_{\sigma(i) j}^{+}}\right)^{k}\right\}^{1 / k}}\right], 1-\frac{1}{1+\left\{\sum_{i=1}^{n} w_{i}\left(\frac{\mu_{\sigma(i) j}}{1-\mu_{\sigma(i) j}}\right)^{k}\right\}^{1 / k}}\right)_{j=1}^{m}$.

The $w_{i}$ and $\sigma(i)$ have usual meanings.

Proof. We can prove it by induction.

For $n=2$, we have

CmPFDOWPAO $\left(A_{1}, A_{2}\right)$

$=\left(\left[1-\frac{1}{1+\left\{w_{1}\left(\frac{\mu_{\sigma(1) j}^{-}}{1-\mu_{\sigma(1) j}^{-}}\right)^{k}+w_{2}\left(\frac{\mu_{\sigma(2) j}^{-}}{1-\mu_{\sigma(2) j}^{-}}\right)^{k}\right\}^{1 / k}}, 1-\frac{1}{1+\left\{w_{1}\left(\frac{\mu_{\sigma(1) j}^{+}}{1-\mu_{\sigma(1) j}^{+}}\right)^{k}+w_{2}\left(\frac{\mu_{\sigma(2) j}^{+}}{1-\mu_{\sigma(2) j}^{+}}\right)^{k}\right\}^{1 / k}}\right]\right.$,

$\left.1-\frac{1}{1+\left\{w_{1}\left(\frac{\mu_{\sigma(1) j}}{1-\mu_{\sigma(1) j}}\right)^{k}+w_{2}\left(\frac{\mu_{\sigma(2) j}}{1-\mu_{\sigma(2) j}}\right)^{k}\right\}^{1 / k}}\right)_{j=1}^{m}$

which is a CmPFN, by definition.

We can grip induction hypothesis. Now we see that

$\operatorname{CmPFDOWPAO}\left(A_{1}, A_{2}, \cdots, A_{n}\right)=w_{1} A_{\sigma(1)} \oplus_{P} w_{2} A_{\sigma(2)} \oplus_{P} \cdots \oplus_{P} w_{n} A_{\sigma(n)}$

$=\left(\left[1-\frac{1}{1+\left\{\sum_{i=1}^{n} w_{i}\left(\frac{\mu_{\sigma(i) j}^{-}}{1-\mu_{\sigma(i) j}^{-}}\right)^{k}\right\}^{1 / k}}, 1-\frac{1}{1+\left\{\Sigma_{i=1}^{n} w_{i}\left(\frac{\mu_{\sigma(i) j}^{+}}{1-\mu_{\sigma(i) j}^{+}}\right)^{k}\right\}^{1 / k}}\right], 1-\frac{1}{1+\left\{\sum_{i=1}^{n} w_{i}\left(\frac{\mu_{\sigma(i) j}}{1-\mu_{\sigma(i) j}}\right)^{k}\right\}^{1 / k}}\right)_{j=1}^{m}$

$=\left(\left[1-\frac{1}{1+\left\{\Sigma_{i=1}^{n-1} w_{i}\left(\frac{\mu_{\sigma(i) j}^{-}}{1-\mu_{\sigma(i) j}^{-}}\right)^{k}\right\}^{1 / k}}, 1-\frac{1}{1+\left\{\Sigma_{i=1}^{n-1} w_{i}\left(\frac{\mu_{\sigma(i) j}^{+}}{1-\mu_{\sigma(i) j}^{+}}\right)^{k}\right\}^{1 / k}}\right], 1-\frac{1}{1+\left\{\sum_{i=1}^{n-1} w_{i}\left(\frac{\mu_{\sigma(i) j}}{1-\mu_{\sigma(i) j}}\right)^{k}\right\}^{1 / k}}\right)_{j=1}^{m}$

$\oplus_{P} w_{n}\left(\left[\mu_{\sigma(n) j}^{-}, \mu_{\sigma(n) j}^{+}\right], \mu_{\sigma(n) j}\right)$

which is surely a CmPFN by induction basis/hypothesis.

We can prove the following properties for CMPFDOWPAO. 
Theorem 14. CmPFDOWPAO ensures its compatibility for ICmPFNs. That is, if $A_{1}, A_{2}, \cdots, A_{n}$ are ICmPFNs, then CmPFDOWPAO $\left(A_{1}, A_{2}, \cdots, A_{n}\right)$ is an ICmPFN.

Proof. The proof is similar to Theorem 9.

Theorem 15 (Idempotency). Let $A_{i}=\left(\left[\mu_{i j}^{-}, \mu_{i j}^{+}\right], \mu_{i j}\right)_{j=1}^{m}, i=1,2, \cdots, n$, be the assemblage of CmPFNs such that $A_{i}=A=\left(\left[\mu_{j}^{-}, \mu_{j}^{+}\right], \mu_{j}\right)_{j=1}^{m}$ for all $i$. Then $\operatorname{CmPFDOWPAO}\left(A_{1}, A_{2}, \cdots\right.$, $\left.A_{n}\right)=A$.

Proof. Consider CmPFDOWPAO $\left(A_{1}, A_{2}, \cdots, A_{n}\right)=w_{1} A_{\sigma(1)} \oplus_{P} w_{2} A_{\sigma(2)} \oplus_{P} \cdots \oplus_{P} w_{n} A_{\sigma(n)}$ $=w_{1} A \oplus_{P} w_{2} A \oplus_{P} \cdots \oplus_{P} w_{n} A$

$$
\begin{aligned}
& =\left(\left[1-\frac{1}{1+\left\{\sum_{i=1}^{n} w_{i}\left(\frac{\mu_{j}^{-}}{1-\mu_{j}^{-}}\right)^{k}\right\}^{1 / k}}, 1-\frac{1}{1+\left\{\Sigma_{i=1}^{n} w_{i}\left(\frac{\mu_{j}^{+}}{1-\mu_{j}^{+}}\right)^{k}\right\}^{1 / k}}\right], 1-\frac{1}{1+\left\{\sum_{i=1}^{n} w_{i}\left(\frac{\mu_{j}}{1-\mu_{j}}\right)^{k}\right\}^{1 / k}}\right)_{j=1}^{m} \\
& =\left(\left[1-\frac{1}{1+\left\{\left(\frac{\mu_{j}^{-}}{1-\mu_{j}^{-}}\right)^{k}\right\}^{1 / k}}, 1-\frac{1}{1+\left\{\left(\frac{\mu_{j}^{+}}{1-\mu_{j}^{+}}\right)^{k}\right\}^{1 / k}}\right], 1-\frac{1}{1+\left\{\left(\frac{\mu_{j}}{1-\mu_{j}}\right)^{k}\right\}^{1 / k}}\right)_{j=1}^{m} \\
& =\left(\left[\mu_{j}^{-}, \mu_{j}^{+}\right], \mu_{j}\right)_{j=1}^{m}=A, \\
& w=\left(w_{i}\right)_{i=1}^{n} \text { being the weight vector. }
\end{aligned}
$$

Theorem 16 (Monotonicity). For the two collections of CmPFNs $A_{i}=\left(\left[\mu_{i j}^{-}, \mu_{i j}^{+}\right], \mu_{i j}\right)_{j=1}^{m}$ and $B_{i}=\left(\left[v_{i j}^{-}, v_{i j}^{+}\right], v_{i j}\right)_{j=1}^{m}, i=1,2, \cdots, n$, with $A_{i} \leq{ }_{P} B_{i}$ for all $i, \operatorname{CmPFDOWPAO}\left(A_{1}, A_{2}, \cdots\right.$, $\left.A_{n}\right) \leq{ }_{P} \operatorname{CmPFDOWPAO}\left(B_{1}, B_{2}, \cdots, B_{n}\right)$.

Proof. Theorem is the same as Theorem 3.

Theorem 17 (Boundedness). Let $A_{i}=\left(\left[\mu_{i j}^{-}, \mu_{i j}^{+}\right], \mu_{i j}\right)_{j=1}^{m}, i=1,2, \cdots, n$, be the collection of CmPFNs. We define $\vee_{P} A_{i}=A^{+}$and $\wedge_{P} A_{i}=A^{-}$. Then

$$
A^{-} \leq_{P} \operatorname{CmPFDOWPAO}\left(A_{1}, A_{2}, \cdots, A_{n}\right) \leq_{P} A^{+}
$$

Proof. Straightforward. To date, we have discussed CmPFDPAO, CmPFDWPAO, and CMPFDOWPAO and related properties for CmPFEs. These operators have their own advantages. However, they have some limitations as well. CmPFDPAO does not work in a weighted environment, CmPFDWPAO weights only $\mathrm{CmPF}$ values, and only ordered positions are weighted under CmPFDOWPAO. To overcome this limitation, we define a new aggregation operator that is a hybrid of CmPFDWPAO and CmPFDOWPAO and will weight $\mathrm{CmPF}$ values as well as their ordered positions.

Definition 16. A cubic m-polar fuzzy Dombi hybrid P-averaging operator (CmPFDHPAO) is a function from n-dimensional CmPF space to CmPF space. If we have a collection of CmPFNs $A_{i}=$ $\left(\left[\mu_{i j}^{-}, \mu_{i j}^{+}\right], \mu_{i j}\right)_{j=1}^{m}, i=1,2, \cdots, n$, then the CmPFDHPAO weighted by $w=\left(w_{1}, w_{2}, \cdots, w_{n}\right)$, $w_{i}>0, \Sigma_{i=1}^{n} w_{i}=1$ is defined as

$$
\operatorname{CmPFDHPAO}\left(A_{1}, A_{2}, \cdots, A_{n}\right)=w_{1} A_{\sigma(1)}^{\prime} \oplus_{P} w_{2} A_{\sigma(2)}^{\prime} \oplus_{P} \cdots \oplus_{P} w_{n} A_{\sigma(n)}^{\prime},
$$

where $A_{i}^{\prime}=n \mho_{i} A_{i} ; n$ is balancing factor, $\mho=\left(\mho_{i}\right)_{i=1}^{n}$ is weight vector for $A_{i=1}^{\prime}$ with the condition $\mho_{i}>0$ and $\Sigma_{i=1}^{n} \mho_{i}=1$. Here, $\sigma$ has usual meanings as in Definition 3.

Interestingly, CmPFDHPAO becomes CmPFDWPAO if we take $w=(1 / n, 1 / n, \cdots$, $1 / n)$, and it becomes CmPFDOWPAO if we take $\mho=(1 / n, 1 / n, \cdots, 1 / n)$. Therefore, 
CIPFDHPAO is the generalized one with CMPFDWPAO and CmPFDOWPAO as its special cases.

\section{CmPF Dombi Averaging Aggregation Operators with R-order}

In this section, we introduce some Dombi R-aggregation operators for CmPF information. We will discuss some properties of these AOs.

Definition 17. For a collection of CmPFNs $A_{1}, A_{2}, \cdots, A_{n}$, the cubic m-polar fuzzy Dombi $R$-averaging operator is defined as

$$
\operatorname{CmPFDRAO}\left(A_{1}, A_{2}, \cdots, A_{n}\right)=A_{1} \oplus_{R} A_{2} \oplus_{R} \cdots \oplus_{R} A_{n} .
$$

Theorem 18. Let $A_{i}=\left(\left[\mu_{i j}^{-}, \mu_{i j}^{+}\right], \mu_{i j}\right)_{j=1}^{m}, i=1,2, \cdots, n$, be the collection of CmPFNs. Then the aggregated value under CmPFDRAO is again a CmPFN and

$\operatorname{CmPFDRAO}\left(A_{1}, A_{2}, \cdots, A_{n}\right)=\left(\left[1-\frac{1}{1+\left\{\sum_{i=1}^{n}\left(\frac{\mu_{i j}^{-}}{1-\mu_{i j}^{-}}\right)^{k}\right\}^{1 / k}}, 1-\frac{1}{1+\left\{\sum_{i=1}^{n}\left(\frac{\mu_{i j}^{+}}{1-\mu_{i j}^{+}}\right)^{k}\right\}^{1 / k}}\right]\right.$,

$\left.\frac{1}{1+\left\{\Sigma_{i=1}^{n}\left(\frac{1-\mu_{i j}}{\mu_{i j}}\right)^{k}\right\}^{1 / k}}\right)_{j=1}^{m}$

Proof. Proof is the same as Theorem 6.

Theorem 19 (Commutative). For any collection of CmPFNs $A_{i}=\left(\left[\mu_{i j}^{-}, \mu_{i j}^{+}\right], \mu_{i j}\right)_{j=1}^{m}, i=$ $1,2, \cdots, n$,

$\operatorname{CmPFDRAO}\left(A_{1}, A_{2}, \cdots, A_{n}\right)=\operatorname{CmPFDRAO}\left(\hat{A}_{1}, \hat{A}_{2}, \cdots, \hat{A}_{n}\right)$, where $\left(\hat{A}_{i}\right)_{i=1}^{n}$ is a permutation of $\left(A_{i}\right)_{i=1}^{n}$.

Proof. Follows from definition.

Definition 18. For a collection of CmPFNs $A_{1}, A_{2}, \cdots, A_{n}$, the Cubic m-Polar Fuzzy Dombi Weighted $R$-Averaging Operator is defined as

$\operatorname{CmPFDWRAO}\left(A_{1}, A_{2}, \cdots, A_{n}\right)=w_{1} A_{1} \oplus_{R} w_{2} A_{2} \oplus_{R} \cdots \oplus_{R} w_{n} A_{n}$, where $w=\left(w_{1}, w_{2}, \cdots\right.$, $\left.w_{n}\right)$ is a weight vector with $\Sigma_{j=1}^{n} w_{j}=1$ and $w_{j}>0$.

Theorem 20. Let $A_{i}=\left(\left[\mu_{i j}^{-}, \mu_{i j}^{+}\right], \mu_{i j}\right)_{j=1}^{m}, i=1,2, \cdots, n$, be the collection of CmPFNs. Then the aggregated value under CmPFDWRAO is again a CmPFN and

$\operatorname{CmPFDWRAO}\left(A_{1}, A_{2}, \cdots, A_{n}\right)$

$=\left(\left[1-\frac{1}{1+\left\{\Sigma_{i=1}^{n} w_{i}\left(\frac{\mu_{i j}^{-}}{1-\mu_{i j}^{-}}\right)^{k}\right\}^{1 / k}}, 1-\frac{1}{1+\left\{\Sigma_{i=1}^{n} w_{i}\left(\frac{\mu_{i j}^{+}}{1-\mu_{i j}^{+}}\right)^{k}\right\}^{1 / k}}\right], \frac{1}{1+\left\{\sum_{i=1}^{n} w_{i}\left(\frac{1-\mu_{i j}}{\mu_{i j}}\right)^{k}\right\}^{1 / k}}\right)_{j=1}^{m}$

Proof. The following properties can be easily proved for CmPFDWRAO.

Theorem 21 (Idempotency). Let $A_{i}=\left(\left[\mu_{i j}^{-}, \mu_{i j}^{+}\right], \mu_{i j}\right)_{j=1}^{m}, i=1,2, \cdots, n$, be the assembly of CmPFNs such that $A_{i}=A=\left(\left[\mu_{j}^{-}, \mu_{j}^{+}\right], \mu_{j}\right)_{j=1}^{m}$. Then, CmPFDWRAO $\left(A_{1}, A_{2}, \cdots, A_{n}\right)=A$.

Theorem 22 (Monotonicity). Let $A_{i}=\left(\left[\mu_{i j}^{-}, \mu_{i j}^{+}\right], \mu_{i j}\right)_{j=1}^{m}$ and $B_{i}=\left(\left[v_{i j}^{-}, v_{i j}^{+}\right], v_{i j}\right)_{j=1}^{m}, i=$ $1,2, \cdots, n$, be the two collections of CmPFNs such that $A_{i} \leq_{R} B_{i}$ for all $i$. Then $\operatorname{CmPFDWRAO}\left(A_{1}, A_{2}, \cdots, A_{n}\right) \leq_{R} \operatorname{CmPFDWRAO}\left(B_{1}, B_{2}, \cdots, B_{n}\right)$. 
Theorem 23 (Boundedness). Let $A_{i}=\left(\left[\mu_{i j}^{-}, \mu_{i j}^{+}\right], \mu_{i j}\right)_{j=1}^{m}, i=1,2, \cdots, n$, be the collection of CmPFNs. We define $\vee_{R} A_{i}=A^{+}$and $\wedge_{R} A_{i}=A^{-}$. Then

$$
A^{-} \leq_{R} \operatorname{CmPFDWRAO}\left(A_{1}, A_{2}, \cdots, A_{n}\right) \leq_{R} A^{+}
$$

Definition 19. Let $A_{1}, A_{2}, \cdots, A_{n}$ be the fabrication of CmPFNs, the Cubic m-Polar Fuzzy Dombi Ordered Weighted $R$-Averaging Operator is defined as

$\operatorname{CmPFDOWRAO}\left(A_{1}, A_{2}, \cdots, A_{n}\right)=w_{1} A_{\sigma(1)} \oplus_{R} w_{2} A_{\sigma(2)} \oplus_{R} \cdots \oplus_{R} w_{n} A_{\sigma(n)}$, where $w=$ $\left(w_{1}, w_{2}, \cdots, w_{n}\right)$ is a weight vector with $\sum_{j=1}^{n} w_{j}=1$ and $w_{j}>0$, and $\sigma(i)$ is a permutation of $(i)_{i=1}^{n}$ dictating $A_{\sigma(1)} \geq_{R} A_{\sigma(2)} \geq_{R} \cdots \geq_{R} A_{\sigma(n)}$.

Theorem 24. Let $A_{i}=\left(\left[\mu_{i j}^{-}, \mu_{i j}^{+}\right], \mu_{i j}\right)_{j=1}^{m}, i=1,2, \cdots, n$, be the knot of CmPFNs. Then, the accumulated value under CmPFDOWRAO is a CmPFN and

$$
\begin{aligned}
& \operatorname{CmPFDOWRAO}\left(A_{1}, A_{2}, \cdots, A_{n}\right) \\
= & \left(\left[1-\frac{1}{1+\left\{\sum_{i=1}^{n} w_{i}\left(\frac{\mu_{\sigma(i) j}^{-}}{1-\mu_{\sigma(i) j}^{-}}\right)^{k}\right\}^{1 / k}}, 1-\frac{1}{1+\left\{\sum_{i=1}^{n} w_{i}\left(\frac{\mu_{\sigma(i) j}^{+}\left(1-\mu_{\sigma(i) j}\right.}{1+k}\right\}^{1 / k}\right.}\right], \frac{1}{1+\left\{\sum_{i=1}^{n} w_{i}\left(\frac{1-\mu_{\sigma(i) j}}{\mu_{\sigma(i) j}}\right)^{k}\right\}^{1 / k}}\right)_{j=1}^{m} .
\end{aligned}
$$

The $w_{i}$ and $\sigma(i)$ have usual meanings.

We can prove the following properties for CMPFDOWRAO.

Theorem 25 (Idempotency). Let $A_{i}=\left(\left[\mu_{i j}^{-}, \mu_{i j}^{+}\right], \mu_{i j}\right)_{j=1}^{m}, i=1,2, \cdots, n$, be the assemblage of CmPFNs such that $A_{i}=A=\left(\left[\mu_{j}^{-}, \mu_{j}^{+}\right], \mu_{j}\right)_{j=1}^{m}$, say, for all $i$. Then, CmPFDOWRAO $\left(A_{1}, A_{2}\right.$, $\left.\cdots, A_{n}\right)=A$.

Theorem 26 (Monotonicity). For any two collections of CmPFNs $A_{i}=\left(\left[\mu_{i j}^{-}, \mu_{i j}^{+}\right], \mu_{i j}\right)_{j=1}^{m}$ and $B_{i}=\left(\left[v_{i j}^{-}, v_{i j}^{+}\right], v_{i j}\right)_{j=1}^{m}, i=1,2, \cdots, n$, with $A_{i} \leq_{R} B_{i}$ for all $i, \operatorname{CmPFDOWRAO}\left(A_{1}, A_{2}, \cdots\right.$, $\left.A_{n}\right) \leq_{R} \operatorname{CmPFDOWRAO}\left(B_{1}, B_{2}, \cdots, B_{n}\right)$.

Theorem 27 (Boundedness). Let $A_{i}=\left(\left[\mu_{i j}^{-}, \mu_{i j}^{+}\right], \mu_{i j}\right)_{j=1}^{m}, i=1,2, \cdots, n$, be the collection of CmPFNs. We define $\vee_{R} A_{i}=A^{+}$and $\wedge_{R} A_{i}=A^{-}$. Then,

$$
A^{-} \leq_{R} \operatorname{CmPFDOWRAO}\left(A_{1}, A_{2}, \cdots, A_{n}\right) \leq_{R} A^{+} .
$$

We have discussed CMPFDRAO, CMPFDWRAO, and CMPFDOWRAO and related properties for CmPFEs. These operators have some limitations already mentioned in Section 3. Therefore, hybridization of CmPFDWRAO and CmPFDOWRAO is mandatory.

Definition 20. A cubic m-polar fuzzy Dombi hybrid R-averaging operator (CmPFDHRAO) is a function CmPFDHRAO : $A^{n} \rightarrow$ A. For CmPFNs $A_{i}=\left(\left[\mu_{i j}^{-}, \mu_{i j}^{+}\right], \mu_{i j}\right)_{j=1}^{m}, i=1,2, \cdots, n$, the CmPFDHRAO weighted by $w=\left(w_{1}, w_{2}, \cdots, w_{n}\right), w_{i}>0, \sum_{i=1}^{n} w_{i}=1$ is defined as

$$
\operatorname{CmPFDHRAO}\left(A_{1}, A_{2}, \cdots, A_{n}\right)=w_{1} A_{\sigma(1)}^{\prime} \oplus_{R} w_{2} A_{\sigma(2)}^{\prime} \oplus_{R} \cdots \oplus_{R} w_{n} A_{\sigma(n)}^{\prime},
$$

where $A_{i}^{\prime}=n \mho_{i} A_{i}, n$ is balancing factor, $\mho=\left(\mho_{i}\right)_{i=1}^{n}$ is weight vector for $A_{i=1}^{\prime}$ with the condition $\mho_{i}>0$ and $\Sigma_{i=1}^{n} \mho_{i}=1$. Here, $\sigma$ is a permutation on $\{1,2, \cdots, n\}$ which dictates $A_{i}^{\prime} s$ in descending order. CmPFDWRAO and CMPFDOWRAO can be observed as special cases of CmPFDHRAO by taking $w=(1 / n, 1 / n, \cdots, 1 / n)$ and $\mho=(1 / n, 1 / n, \cdots, 1 / n)$, respectively. 


\section{MCDM towards the Circular Economy}

In this section, we develop a multi-criteria decision-making (MCDM) technique under cubic m-polar fuzzy information and its application to circular economy (CE). The circular economy (CE) is currently a common concept advocated by many white collar countries and many businesses around the world. However, the science and research fabric of the CE theory is simplistic and unfocused. CE, no doubt, is the best alternative of the linear economy, but its applicability is reduced until its complexities are alleviated.

The word "circular economy" has both a descriptive and linguistic sense. In latter sense, it is opposite to CLE, which is characterized as the conversion of natural resources into waste through processing. Such waste generation leads to environmental destruction by depleting natural resources and increasing pollution. The word "linear economy" has been extensively used since the birth of "circular economy", which is an economy with a minor or no net impact on the climate. It is intended to restore any harm to the resources while guaranteeing little waste during the entire manufacturing period. There are many biochemical and geochemical cycles on the earth that inspired the idea of CE. For instance, water evaporates from the earth water bodies, forms rain drops, comes back to the earth and again becomes a part of the rivers, seas, oceans etc. Similar biogeochemical cycles can be observed on the earth. Each cycle has its own time perio, e.g., water cycle takes about 9 to 10 days, carbon dioxide takes 4.5 years, oxygen in the atmosphere takes 3.8 years to complete. Such biogeochemical cycles in nature are the reason of the existence of humankind on the earth. The water cycle is shown in the Figure 1.

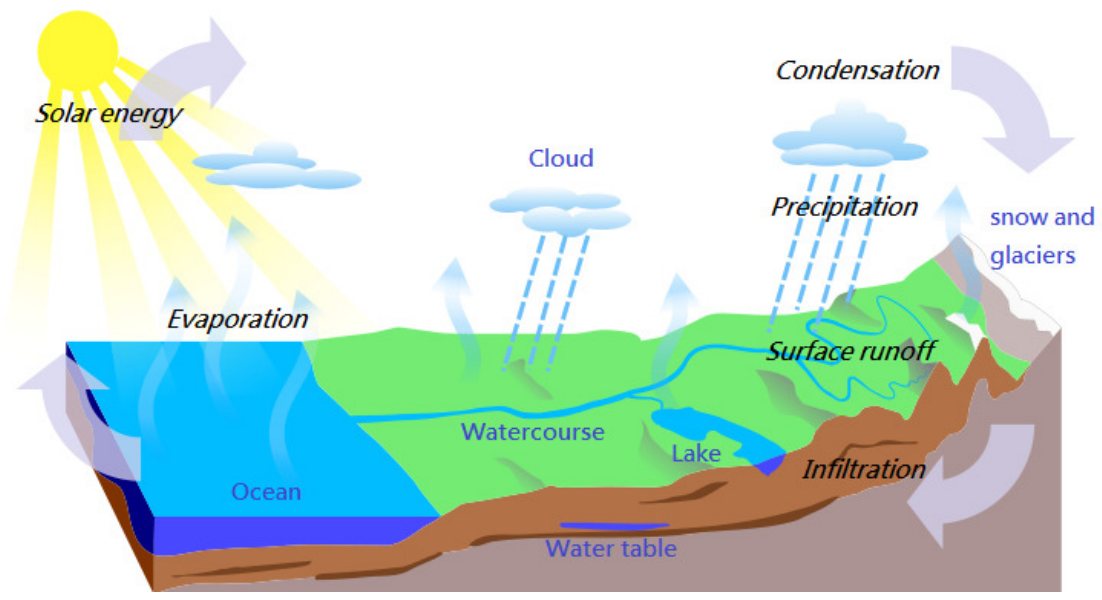

Figure 1. Water cycle.

The practice of CLE has altered almost every cycle. In order to safeguard the existing cycles in nature, it is advisable to promote CE. What makes CE implementable are recycling, repairing, recovering, regenerating etc. The most important and achievable of these is recycling. Recycling refers to the process of transferring sludge into new materials and products. This definition also includes energy recovery from waste materials. The ability of a material to reclaim the properties it had in its pure state determines its renewability. Recycling can help to reduce waste from genuinely useful products while also lowering the cost of new raw materials. Recycling is a central facet of current waste diversion and is the third level of the "Reduce, Reuse, and Recycle" hierarchy. The materials that can be recycled include glass, cardboard, plastic, paper, tires, textiles, metals, and electronics. Each of these are recycled in a unique way. For example, if we focus on recycling plastic 
materials, three major processes are frankly useful depending on the type of the plastic under consideration.

- Chemical recycling

- Heat compression

- Mechanical recycling

Chemical recycling.

Polymers are a special type of plastic manufactured chemically. These are basically complex chemical combinations of monomers. A wide range of polymers may be converted back into monomers. PET, for example, is a well-known polymer. It is converted to dialkyl terephthalate if treated with alcohol and an appropriate catalyst. The terephthalate diester is then treated with ethylene glycol, yielding a pure form of a new polymer known as polyester polymer. As a result, various types of plastics can be effectively recycled using chemical methods.

Heat compression.

In heat compression, plastics of all sorts are mixed together, compressed, and rolled in a large heated and rolling tumbler. This is a beneficial way to recycle the plastic. However, the tumblers involved render this process uneconomical because it again involves the usage of natural resources like coal, oil, gas etc. to rotate the tumbler and for compression purpose. Therefore, this process bears some criticism.

Mechanical recycling.

Some plastics are melted down to shape new objects. For example, PET plastic can be processed into polyester, which is intended for clothes. A downside of this recycling method is that the polymer's molecular mass can alter with each remelt, and the amounts of fish waste in the plastic can increase.

Plastic recycling process can be categorized into three steps: Collection, Reprocessing, and Production. The main contribution to these three steps mainly comes from collectors, suppliers, sorters, and recyclers.

Collection.

Recycling operation starts with the contribution of garbage pickers and dealers. A recycling organization involves a network of formal collectors participating in collecting and sorting of recyclable plastic materials. Garbage pickers include two categories: those who work legally with a company and those who are not bound to any specific organization. The second type of picker is critical to the industry. They work independently, inconsistently, and they do not bother the liability of the industry. However, the plastic recycling industry relies on them, to some extent, indirectly. Due to their informal and erratic work hours, recyclables are not routinely supplied to recyclers, and hence it is not beneficial to the industry. Therefore, to secure a reliable position in the market, a recycler must minimize its dependance on critical pickers.

Reprocessing.

After the waste plastic is collected, it is supplied to the recycling plants by the dealers, where it undergoes one of the above mentioned recycling process followed by resorting. Resorting is indispensable for the circular economy. Some plastic materials are economically recycled under heat compression, some using chemical and some mechanical methods according to their resin type. This categorization is accomplished in reprocessing. Production. 
When sorted, plastic recyclables are eviscerated for mechanical, chemical, or heated recycling. The pieces are shredded and treated in order to extract impurities such as paper annotations. The material is melted or chemically treated to produce other items.

In order to make unanimous, clever and well-suited decisions in cubic m-polar premises, we propose an extended SIR method that is based on coding superiority index/flow and inferiority index/flow and that dictates the affirmation of the most desired/ideal option in contrast. We first give an algorithm/technique and then apply it to deal with the problem of selecting the most effective recycling plant that can help transform a CLE into a CE in an ideal way. An extended superiority and inferiority ranking (SIR) technique under CmPFSs is developed in the following Algorithm 1.

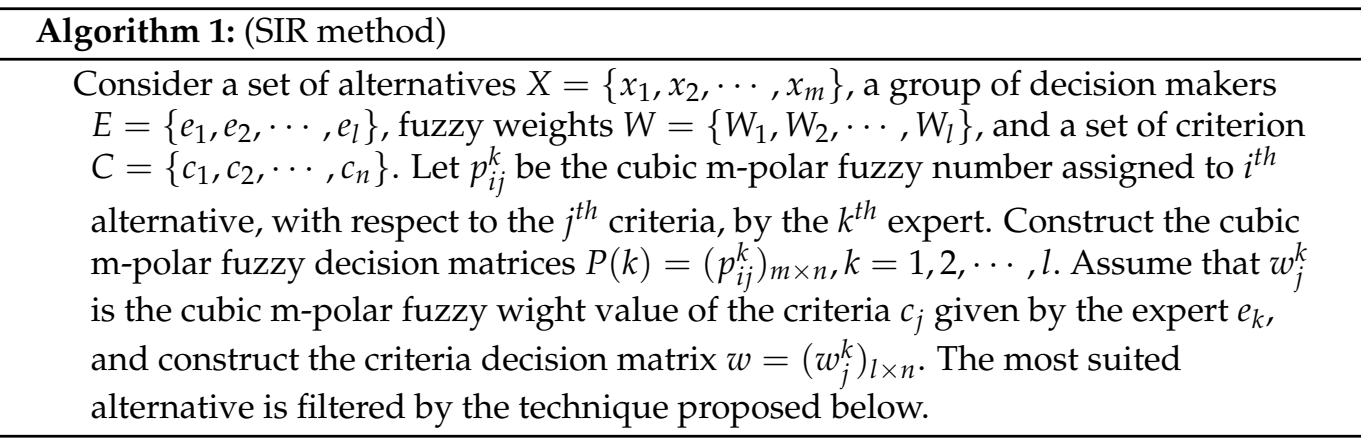

Step 1: Determine the relative proximity coefficient by the formula

$$
\xi_{k}=\frac{d\left(W_{k}, W^{-}\right)}{d\left(W_{k}, W^{-}\right)+d\left(W_{k}, W^{+}\right)}
$$

where $W^{-}$and $W^{+}$denote, respectively, the P-minimum and P-maximum. It immediately follows from the formula that if $W_{k} \rightarrow W^{+}$, then $\xi_{k} \rightarrow 1$. Similarly, if $W_{k} \rightarrow W^{-}$, then $\xi_{k} \rightarrow 0$. Furthermore, $0 \leq \xi_{k} \leq 1$.

Step 2: If the $\xi_{k}$ are in normal form, that is, if they sum up to unity, name them as $\zeta_{k}$. Otherwise, normalize them by the formula

$$
\zeta_{k}=\frac{\xi_{k}}{\sum_{k=1}^{l} \xi_{k}}
$$

In this way, we obtain normalized estimation degrees $\zeta=\left(\zeta_{1}, \zeta_{2}, \cdots, \zeta_{l}\right)$.

Step 3: Obtain the combined cubic m-polar decision matrix $p=\left(\bar{p}_{i j}\right)_{m \times n}$ and the weight vector $\bar{w}=\left(\bar{w}_{j}\right)_{j=1}^{n}$ using one of the proposed operators, where

$$
\begin{aligned}
& \bar{p}_{i j}=\operatorname{CmPFDWPAO}\left(p_{i j}^{1}, p_{i j}^{2}, \cdots, p_{i j}^{l}\right)=\operatorname{CmPFDWPAO}\left(p_{i j}^{k}\right)_{k=1}^{l}, \\
& \bar{w}_{j}=\operatorname{CmPFDWPAO}\left(w_{j}^{1}, w_{j}^{2}, \cdots, w_{j}^{l}\right)=\operatorname{CmPFDWPAO}\left(w_{j}^{k}\right)_{k=1}^{l} .
\end{aligned}
$$

(In the end, we give a comparison analysis of CmPFDWPAO with the other proposed operators.)

Step 4: Construct the relative performance relation

$$
f_{i j}=\frac{d\left(\bar{p}_{i j}, \bar{p}^{-}\right)}{d\left(\bar{p}_{i j}, \bar{p}^{-}\right)+d\left(\bar{p}_{i j}, \bar{p}^{+}\right)},
$$


where $\bar{p}^{+}=\max _{i} \bar{p}_{i j}$ and $\bar{p}^{-}=\min _{i} \bar{p}_{i j}$. Clearly, if $\bar{p}_{i j} \rightarrow \bar{p}^{-}$, then $f_{i j} \rightarrow 0$ and if $\bar{p}_{i j} \rightarrow \bar{p}^{+}$, then $f_{i j} \rightarrow 1$. Furthermore, $0 \leq f_{i j} \leq 1$.

After this, construct superiority matrix $S=\left(S_{i j}\right)_{m \times n}$ and inferiority matrix $I=$ $\left(I_{i j}\right)_{m \times n}$, where

$$
S_{i j}=\sum_{t=1}^{m} \phi\left(f_{i j}-f_{t j}\right)
$$

and

$$
I_{i j}=\sum_{t=1}^{m} \phi\left(f_{t j}-f_{i j}\right) .
$$

$\phi(x)$ being the threshold function given by

$$
\phi(x)= \begin{cases}0.01 & 0<x<1 \\ 0.00 & x \leq 0 \text { or } x \geq 1 .\end{cases}
$$

Step 5: The superiority index and inferiority index can be calculated, respectively, as follows.

$$
\phi^{>}\left(x_{i}\right)=\operatorname{CmPFDWPAO} S_{S_{i j}}\left(\bar{w}_{j}\right)_{j=1}^{n}=\left(S_{i 1} \bar{w}_{1} \oplus_{P} S_{i 2} z \bar{w}_{2} \oplus_{P} \cdots \oplus_{P} S_{i n} \bar{w}_{n}\right),
$$

and

$$
\phi^{<}\left(x_{i}\right)=\operatorname{CmPFDWPAO} I_{i j}\left(\bar{w}_{j}\right)_{j=1}^{n}=\left(I_{i 1} \bar{w}_{1} \oplus_{P} I_{i 2} \bar{w}_{2} \oplus_{P} \cdots \oplus_{P} I_{i n} \bar{w}_{n}\right) .
$$

Step 6: Calculate the score functions of $\phi^{<}\left(x_{i}\right)$ and $\phi^{>}\left(x_{i}\right)$, for all $i=1,2, \cdots, m$, using the Formula (1).

Step 7: Find the superiority flow and inferiority flow according to the following rules. Superiority Flow Rules (SFRs)

- $x_{i}>x_{t}$ if $S\left(\phi^{>}\left(x_{i}\right)\right)>S\left(\phi^{>}\left(x_{t}\right)\right)$ and $S\left(\phi^{<}\left(x_{i}\right)\right)<S\left(\phi^{<}\left(x_{t}\right)\right)$,

- $\quad x_{i}>x_{t}$ if $S\left(\phi^{>}\left(x_{i}\right)\right)>S\left(\phi^{>}\left(x_{t}\right)\right)$ and $S\left(\phi^{<}\left(x_{i}\right)\right)=S\left(\phi^{<}\left(x_{t}\right)\right)$,

- $x_{i}>x_{t}$ if $S\left(\phi^{>}\left(x_{i}\right)\right)=S\left(\phi^{>}\left(x_{t}\right)\right)$ and $S\left(\phi^{<}\left(x_{i}\right)\right)<S\left(\phi^{<}\left(x_{t}\right)\right)$,

Inferiority Flow Rules (SFRs)

- $x_{i}<x_{t}$ if $S\left(\phi^{>}\left(x_{i}\right)\right)<S\left(\phi^{>}\left(x_{t}\right)\right)$ and $S\left(\phi^{<}\left(x_{i}\right)\right)>S\left(\phi^{<}\left(x_{t}\right)\right)$,

- $x_{i}<x_{t}$ if $S\left(\phi^{>}\left(x_{i}\right)\right)<S\left(\phi^{>}\left(x_{t}\right)\right)$ and $S\left(\phi^{<}\left(x_{i}\right)\right)=S\left(\phi^{<}\left(x_{t}\right)\right)$,

- $\quad x_{i}<x_{t}$ if $S\left(\phi^{>}\left(x_{i}\right)\right)=S\left(\phi^{>}\left(x_{t}\right)\right)$ and $S\left(\phi^{<}\left(x_{i}\right)\right)>S\left(\phi^{<}\left(x_{t}\right)\right)$.

Step 8: SF rules coupled with IF rules can filter the optimal alternative.

\subsection{Numerical Example}

The evidence gained in tandem with the circular recycling curriculum is used for the purpose of elucidating model implementation in response to mutually beneficial channels of the program and the towns where it functions and identifies compassionate economic policies for foragers for recyclable materials. A city mayor plans to initiate the practice of $\mathrm{CE}$ in their city. The first step for this purpose is to install a recycling plant. The mayor hires three economists $e_{1}, e_{2}, e_{3}$ and assigns them the credibility weights $W_{1}, W_{2}, W_{3}$ (shown in Table 1). They chose three companies/recycling plants $x_{1}, x_{2}$ and $x_{3}$, which are currently contributing to $\mathrm{CE}$ in certain areas. (Note: We are restricting ourselves to three alternatives and three criteria because our intention is to propose a mathematical model for selecting an optimal recycling plant. The same model is efficient for a big data). Each of the three companies claims that it is the best option for recycling plastic materials, rubber wastes, 
glass wastes, etc. These companies recycle the things in three steps "collection, reprocessing and production". The main problem is to filter the best plant to be installed in the city. The efficiency of each plant is observed on the basis of three criteria shown in Table 2. Their individual assessment turn out to be cubic m-polar fuzzy matrices (shown in Tables 3-5). Step 1: Find $W^{-}=\langle[0.10,0.30],[0.20,0.70],[0.30,0.80], 0.12,0.11,0.50\rangle$ and $W^{+}=\langle[0.80,0.90],[0.83,0.91],[0.85,0.93], 0.90,0.81,0.76\rangle$. Utilize the Formula (3) to calculate

$d\left(W_{1}, W^{-}\right)=0.49852 ; d\left(W_{2}, W^{-}\right)=0.727081 ; d\left(W_{3}, W^{-}\right)=0.947559 ;$ $d\left(W_{1}, W^{+}\right)=0.856648 ; d\left(W_{2}, W^{+}\right)=0.847022 ; d\left(W_{3}, W^{+}\right)=0.539338$.

Calculate the relative proximity coefficients (using Formula (4))

$$
\xi=(0.3679,0.4619,0.6373) .
$$

Step 2: Normalize the estimated proximity degree (using the Formula (5))

$$
\zeta=(0.2508,0.3148,0.4344) .
$$

Step 3: Aggregate the cubic m-polar fuzzy decision information (for $\mathrm{k}=4$ ), provided by the three economists, to figure out the joint information (given in Table 6). The identified criterions are given in Table 7.

Furthermore, obtain the unanimous criteria weights using Equation (7),

$$
\begin{aligned}
\bar{w}_{1} & =\langle[0.28,0.589],[0.32,0.717],[0.329,0.821], 0.346,0.749,0.208\rangle \\
\bar{w}_{2} & =\langle[0.161,0.72],[0.255,0.711],[0.436,0.47], 0.159,0.649,0.325\rangle \\
\bar{w}_{3} & =\langle[0.143,0.532],[0.448,0.77],[0.85,0.976], 0.13,0.145,0.878\rangle .
\end{aligned}
$$

Step 4: The relative performance matrix (using the Formula (8)) is given by

$$
\left(f_{i j}\right)=\left(\begin{array}{lll}
0.604 & 0.289 & 0.300 \\
0.311 & 0.615 & 0.796 \\
0.608 & 0.456 & 0.106
\end{array}\right) .
$$

Construct the superiority and inferiority decision matrices using the Equations (9) and (10), respectively.

$$
S=\left(\begin{array}{lll}
0.01 & 0.00 & 0.01 \\
0.00 & 0.02 & 0.02 \\
0.02 & 0.01 & 0.00
\end{array}\right)
$$

and

$$
I=\left(\begin{array}{lll}
0.01 & 0.02 & 0.01 \\
0.02 & 0.00 & 0.00 \\
0.00 & 0.01 & 0.02
\end{array}\right) .
$$

Steps 5, 6: The superiority and inferiority indices of the alternatives and their respective score functions are given in Tables 8 and 9 .

Step 7: The superiority flow is given by

$$
x_{2}>x_{1}>x_{3}
$$


and the inferiority flow is given by

$$
x_{2}>x_{1}>x_{3}
$$

Step 8: Both the superiority and inferiority flow agree at the optimal alternative $x_{2}$.

Table 1. Credibility weights.

\section{Economists}

$e_{1}$

$e_{2}$

$e_{3}$

\section{Weights}

$W_{1}=\langle[0.20,0.30],[0.50,0.70],[0.45,0.93], 0.61,0.11,0.50\rangle$

$W_{2}=\langle[0.80,0.90],[0.83,0.91],[0.85,0.80], 0.12,0.30,0.70\rangle$

$W_{3}=\langle[0.10,0.80],[0.20,0.75],[0.30,0.85], 0.90,0.81,0.76\rangle$ 
Table 2. Criteria weights assigned by the economists.

$c_{1}$ $\langle[0.27,0.57],[0.30,0.75],[0.32,0.80], 0.30,0.70,0.19\rangle$
$\langle[0.29,0.60],[0.32,0.72],[0.29,0.78], 0.33,0.73,0.17\rangle$
$\langle[0.28,0.59],[0.33,0.67],[0.35,0.84], 0.37,0.77,0.23\rangle$ 西

\begin{tabular}{|c|c|c|c|}
\hline & $c_{1}$ & $c_{2}$ & $c_{3}$ \\
\hline$e_{1}$ & $\langle[0.27,0.57],[0.30,0.75],[0.32,0.80], 0.30,0.70,0.19\rangle$ & $\langle[0.14,0.70],[0.24,0.67],[0.40,0.45], 0.14,0.67,0.31\rangle$ & $\langle[0.11,0.55],[0.44,0.79],[0.87,0.95], 0.10,0.10,0.90\rangle$ \\
\hline$e_{2}$ & $\langle[0.29,0.60],[0.32,0.72],[0.29,0.78], 0.33,0.73,0.17\rangle$ & $\langle[0.16,0.74],[0.27,0.70],[0.46,0.49], 0.17,0.62,0.34\rangle$ & $\langle[0.13,0.54],[0.47,0.77],[0.85,0.93], 0.13,0.14,0.83\rangle$ \\
\hline$e_{3}$ & $\langle[0.28,0.59],[0.33,0.67],[0.35,0.84], 0.37,0.77,0.23\rangle$ & $\langle[0.17,0.71],[0.25,0.73],[0.43,0.47], 0.16,0.65,0.32\rangle$ & $\langle[0.16,0.51],[0.43,0.75],[0.83,0.98], 0.14,0.16,0.87\rangle$ \\
\hline
\end{tabular}

Table 3. Decision matrix by $e_{1}$.

\begin{tabular}{|c|c|c|c|}
\hline$e_{1}$ & $c_{1}$ & $c_{2}$ & $c_{3}$ \\
\hline$x_{1}$ & $\langle[0.60,0.70],[0.50,0.80],[0.40,0.90], 0.40,0.90,0.10\rangle$ & $\langle[0.70,0.90],[0.40,0.70],[0.50,0.80], 0.10,0.20,0.30\rangle$ & $\langle[0.45,0.55],[0.55,0.75],[0.75,0.85], 0.90,0.10,0.70\rangle$ \\
\hline$x_{2}$ & $\langle[0.60,0.80],[0.50,0.70],[0.30,0.60], 0.45,0.85,0.15\rangle$ & $\langle[0.60,0.84],[0.43,0.74],[0.55,0.83], 0.12,0.23,0.34\rangle$ & $\langle[0.43,0.54],[0.54,0.77],[0.85,0.93], 0.88,0.13,0.73\rangle$ \\
\hline$x_{3}$ & $\langle[0.55,0.70],[0.60,0.75],[0.35,0.80], 0.43,0.83,0.25\rangle$ & $\langle[0.66,0.88],[0.39,0.79],[0.60,0.87], 0.16,0.28,0.32\rangle$ & $\langle[0.41,0.55],[0.57,0.75],[0.78,0.81], 0.87,0.14,0.71\rangle$ \\
\hline
\end{tabular}

Table 4. Decision matrix by $e_{2}$.

\begin{tabular}{|c|c|c|c|}
\hline$e_{2}$ & $c_{1}$ & $c_{2}$ & $c_{3}$ \\
\hline$x_{1}$ & $\langle[0.59,0.71],[0.49,0.81],[0.39,0.91], 0.37,0.87,0.08\rangle$ & $\langle[0.69,0.91],[0.39,0.71],[0.49,0.81], 0.07,0.17,0.27\rangle$ & $\langle[0.44,0.56],[0.54,0.76],[0.74,0.86], 0.87,0.13,0.67\rangle$ \\
\hline$x_{2}$ & $\langle[0.59,0.81],[0.49,0.71],[0.29,0.61], 0.42,0.83,0.13\rangle$ & $\langle[0.59,0.85],[0.42,0.75],[0.54,0.84], 0.09,0.21,0.32\rangle$ & $\langle[0.42,0.55],[0.53,0.78],[0.84,0.94], 0.88,0.13,0.73\rangle$ \\
\hline$x_{3}$ & $\langle[0.53,0.72],[0.58,0.77],[0.33,0.82], 0.38,0.80,0.22\rangle$ & $\langle[0.64,0.90],[0.37,0.81],[0.59,0.89], 0.13,0.25,0.29\rangle$ & $\langle[0.39,0.57],[0.55,0.77],[0.76,0.83], 0.84,0.11,0.68\rangle$ \\
\hline
\end{tabular}

Table 5. Decision matrix by $e_{3}$.

\begin{tabular}{|c|c|c|c|}
\hline$e_{3}$ & $c_{1}$ & $c_{2}$ & $c_{3}$ \\
\hline$x_{1}$ & $\langle[0.64,0.66],[0.54,0.76],[0.44,0.86], 0.38,0.89,0.13\rangle$ & $\langle[0.73,0.87],[0.43,0.66],[0.53,0.78], 0.12,0.21,0.30\rangle$ & $\langle[0.49,0.52],[0.59,0.71],[0.79,0.82], 0.92,0.17,0.70\rangle$ \\
\hline$x_{2}$ & $\langle[0.64,0.77],[0.55,0.65],[0.33,0.57], 0.47,0.85,0.17\rangle$ & $\langle[0.65,0.82],[0.47,0.70],[0.50,0.80], 0.14,0.25,0.35\rangle$ & $\langle[0.40,0.51],[0.58,0.73],[0.89,1.00], 0.90,0.17,0.73\rangle$ \\
\hline$x_{3}$ & $\langle[0.58,0.68],[0.63,0.73],[0.38,0.79], 0.43,0.83,0.24\rangle$ & $\langle[0.68,0.86],[0.42,0.76],[0.65,0.83], 0.17,0.29,0.33\rangle$ & $\langle[0.44,0.53],[0.59,0.73],[0.75,0.80], 0.87,0.18,0.70\rangle$ \\
\hline
\end{tabular}

Table 6. Combined/Aggregated decision matrix.

$c_{1}$

\begin{tabular}{ll}
\hline$x_{1}$ & $\langle[0.62,0.69],[0.52,0.79],[0.42,0.9], 0.38,0.89,0.11\rangle$ \\
$x_{2}$ & $\langle[0.62,0.79],[0.52,0.69],[0.31,0.59], 0.45,0.84,0.16\rangle$
\end{tabular}

$x_{3}\langle[0.56,0.70],[0.61,0.75],[0.36,0.80], 0.42,0.82,0.24\rangle$ $c_{2}$

$\langle[0.71,0.90],[0.41,0.69],[0.51,0.80], 0.11,0.20,0.29\rangle$ $\langle[0.63,0.84],[0.45,0.73],[0.53,0.82], 0.13,0.24,0.44\rangle$ $\langle[0.67,0.88],[0.40,0.80],[0.63,0.87], 0.16,0.27,0.31\rangle$ $c_{3}$

$\langle[0.47,0.54],[0.57,0.74],[0.77,0.85], 0.91,0.15,0.69\rangle$ $\langle[0.42,0.53],[0.56,0.76],[0.87,1.00], 0.89,0.15,0.73\rangle$ $\langle[0.42,0.55],[0.58,0.75],[0.76,0.82], 0.86,0.16,0.69\rangle$ 
Table 7. Identified criterions.

\begin{tabular}{ll}
\hline$c_{1}$ & Global Warming Mitigation \\
\hline$c_{2}$ & Friendly to the environment \\
$c_{3}$ & Low energy consumption \\
\hline
\end{tabular}

Table 8. CmPF-SI with their scores.

\begin{tabular}{ccc}
\hline Alternatives & Superiority Indices & Scores \\
\hline$x_{1}$ & $\langle[0.11,0.33],[0.209,0.532],[0.642,0.928], 0.144,0.486,0.695\rangle$ & 0.216 \\
$x_{2}$ & $\langle[0.075,0.494],[0.235,0.573],[0.681,0.939], 0.072,0.410,0.730\rangle$ & 0.297 \\
$x_{3}$ & $\langle[0.128,0.459],[0.155,0.511],[0.208,0.633], 0.166,0.533,0.137\rangle$ & 0.210 \\
\hline
\end{tabular}

Table 9. CmPF-II with their scores.

\begin{tabular}{ccc}
\hline Alternatives & Superiority Indices & Scores \\
\hline$x_{1}$ & $\langle[0.113,0.496],[0.211,0.555],[0.642,0.928], 0.145,0.502,0.695\rangle$ & 0.268 \\
$x_{2}$ & $\langle[0.128,0.35],[0.15,0.489],[0.156,0.633], 0.166,0.529,0.09\rangle$ & 0.211 \\
$x_{3}$ & $\langle[0.068,0.453],[0.234,0.566],[0.681,0.939], 0.064,0.369,0.73\rangle$ & 0.277 \\
\hline
\end{tabular}

\subsection{Comparison Analysis}

In Table 10, we compare suggested aggregation operators with some existing operators to examine the harmony of the proposed model with previous existing operators. The analysis provided therein demonstrates that our proposed model is compatible with those already in the literature. The proposed operators make a credible and legitimate contribution to dealing with uncertainties by utilizing cubic m-polar fuzzy information.

Table 10. Comparative analysis of the proposed operators and existing ones.

\begin{tabular}{lcc}
\hline Method & Ranking of Alternatives & The Optimal Alternative \\
\hline PFDOWA (Jana [30]) & $x_{2} \succ x_{3} \succ x_{4}$ & $x_{2}$ \\
PFDHWA (Jana [30]) & $x_{2} \succ x_{3} \succ x_{1}$ & $x_{2}$ \\
PFOWA (Garg [24]) & $x_{2} \succ x_{1} \succ x_{3}$ & $x_{2}$ \\
PFHA (Garg [24]) & $x_{2} \succ x_{1} \succ x_{3}$ & $x_{2}$ \\
CqROFBM (Liu et al. [41]) & $x_{2} \succ x_{3} \succ x_{4}$ & $x_{2}$ \\
IFEIO (Liu and Wang [42]) & $x_{2} \succ x_{4} \succ x_{1}$ & $x_{2}$ \\
CMPFWAO (Riaz and Hashmi [35]) & $x_{2} \succ x_{1} \succ x_{4}$ & $x_{2}$ \\
CMPFOWAO (Riaz and Hashmi [35]) & $x_{2} \succ x_{1} \succ x_{4}$ & $x_{2}$ \\
CMPFHAO (Riaz and Hashmi [35]) & $x_{2} \succ x_{1} \succ x_{4}$ & $x_{2}$ \\
CmPFDPAO (Proposed) & $x_{2} \succ x_{1} \succ x_{4}$ & $x_{2}$ \\
CmPFDRAO (Proposed) & $x_{2} \succ x_{1} \succ x_{4}$ & $x_{2}$ \\
CmPFDWRAO (Proposed) & $x_{2} \succ x_{1} \succ x_{4}$ & $x_{2}$ \\
CmPFDOWPAO (Proposed) & $x_{2} \succ x_{1} \succ x_{4}$ & $x_{2}$ \\
CmPFDOWRAO (Proposed) & $x_{2} \succ x_{1} \succ x_{4}$ & $x_{2}$ \\
CmPFDHPAO (Proposed) & $x_{2} \succ x_{1} \succ x_{4}$ & $x_{2}$ \\
CmPFDHRAO (Proposed) & $x_{2} \succ x_{1} \succ x_{4}$ & $x_{2}$ \\
\hline
\end{tabular}

\section{Conclusions}

A cubic m-polar fuzzy set (CmPFS) is a powerful model for dealing with various uncertainties in multi-criteria decision making (MCDM) problems. A cubic set (CS) can express vague information using two components: one is a fuzzy interval and the other is a fuzzy number. While an m-polar fuzzy set (mPFS) assigns $m$ degrees to each alternative in the discourse universe. We focus on CmPFS, which is more efficient to address uncertainties in the multi polar information with a group of $m$ fuzzy intervals and $m$ fuzzy 
numbers. We investigate some new aspects and consequences of CmPFSs. We define score and accuracy functions to find the ranking of alternatives/objects in MCDM. Additionally, we introduced some new operations, like addition, scalar/usual multiplication and power, under Dombi's t-conorm and t-norm. We developed several new aggregation operators (AOs) named cubic m-polar fuzzy Dombi P-averaging operator (CmPFDPAO), cubic m-polar fuzzy Dombi R-averaging operator (CmPFDRAO), cubic m-polar fuzzy Dombi weighted P-averaging operator (CmPFDWPAO), cubic m-polar fuzzy Dombi weighted R-averaging operator (CmPFDWRAO), cubic m-polar fuzzy Dombi ordered weighted P-averaging operator (CmPFDOWPAO), cubic m-polar fuzzy Dombi ordered weighted R-averaging operator (CmPFDOWRAO), cubic m-polar fuzzy Dombi hybrid P-averaging Operator (CmPFDHPAO) and cubic m-polar fuzzy Dombi hybrid R-averaging operator (CmPFDHRAO). Certain properties, like, monotonicity, commutativity, idempotency, and boundedness are explored. An advanced superiority and inferiority ranking (SIR) technique is developed to deal with the problem of conversion from linear economy to circular economy. Lastly, a comparison analysis of proposed methodology with some other existing methods is also given.

Author Contributions: M.R., D.P., and M.A.K., conceived and worked together to achieve this manuscript, D.P., and M.A. construct the ideas and algorithms for data analysis and design the model of the manuscript, M.R., M.A.K., and M.A. processed the data collection and wrote the paper. All authors have read and agreed to the published version of the manuscript.

Funding: Abha 61413, Saudi Arabia, research groups program under grant number R.G. P-2/29/42. Institutional Review Board Statement: Not applicable.

Informed Consent Statement: Not applicable.

Data Availability Statement: Not applicable.

Acknowledgments: The authors extend their appreciation to the Deanship of Scientific Research at King Khalid University, Abha 61413, Saudi Arabia for funding this work through research groups program under grant number R.G. P-2/29/42.

Conflicts of Interest: The authors declare no conflict of interest.

\section{References}

1. Korhonen, J.; Honkasalo, A.; Seppala, J. Circular Economy: The Concept and its Limitations. Ecol. Econ. 2018, 143, 37-46. [CrossRef]

2. Morseletto, P. Targets for a circular economy. Resour. Conserv. Recycl. 2019, 153, 1-12. [CrossRef]

3. Saidani, M.; Yannou, B.; Leroy, Y.; Cluzel, F.; Kendall, A. A taxonomy of circular economy indicators. J. Clean. Prod. 2019, 207, 542-559. [CrossRef]

4. Ghosh, S.K. Introduction to circular economy and summary analysis of chapters. In Circular Economy: Global Perspective; Springer: Berlin/Heidelberg, Germany, 2019; pp. 1-23. [CrossRef]

5. Zhao, H.; Guo, S.; Zhao, H. Comprehensive benefit evaluation of eco-industrial parks by employing the best-worst method based on circular economy and sustainability. Environ. Dev. Sustain. 2018, 20, 1229-1253. [CrossRef]

6. Sanchez, F.A.C.; Boudaoud, H.; Camargo, M.; Pearce, J.M. Plastic recycling in additive manufacturing: A systematic literature review and opportunities for the circular economy. J. Clean. Prod. 2020, 264, 121602. [CrossRef]

7. Yang, X.; Niu, D.; Chen, M.; Wang, K.; Wang, Q.; Xu, X. An operation benefit analysis and decision model of thermal power enterprises in china against the background of large-scale new energy consumption. Sustainability 2020, 12, 4642. [CrossRef]

8. Bacher, J.; Pihkola, H.; Kujanpaa, L.; Mroueh, U. Advancing the circular economy through group decision-making and stakeholder involvement. Detritus 2018, 4, 22-35. [CrossRef] 
9. Fidelis, R.; Ferreira, M.A.; Colmenero, J.C. Selecting a location to install a plastic processing center: Network of recycling cooperatives. Resour. Conserv. Recycl. 2015, 103, 1-8. [CrossRef]

10. Zadeh, L.A. Fuzzy sets. Inf. Control. 1965, 8, 338-353. [CrossRef]

11. Atanassov, K.T. Intuitionistic fuzzy sets. Fuzzy Sets Syst. 1986, 20, 87-96. [CrossRef]

12. Molodtsov, D. Soft set theory-first results. Comput. Math. Appl. 1999, 37, 19-31. [CrossRef]

13. Zhang, W.R. Bipolar fuzzy sets and relations: a computational framework for cognitive modeling and multiagent decision analysis, NAFIPS/IFIS/NASA94. In Proceedings of the First International Joint Conference of The North American Fuzzy Information Processing Society Biannual Conference. The Industrial Fuzzy Control and Intellige, San Antonio, TX, USA, 18-21 December 1994 ; pp. 305-309.

14. Zhang, W.R. Bipolar fuzzy sets. In Proceedings of the IEEE Inrenational Conference on Fuzzy Systems, Anchorage, AK, USA, 4-9 May 1998; pp. 835-840.

15. Smarandache, F. A Unifying Field in Logics. Neutrosophy: Neutrosophic Probability, Set and Logic; American Research Press: Rehoboth, DE, USA, 1999

16. Smarandache F. Neutrosophic set-a generalization of the intuitionistic fuzzy set. Int. J. Pure Appl. Math. 2005, 24, $287-297$.

17. Cuong, B.C. Picture fuzzy sets. Comput. Sci. Cybern. 2014, 30, 409-420.

18. Yager, R.R. Pythagorean fuzzy subsets. In Proceedings of the Joint IFSA World Congress and NAFIPS Annual Meeting, Edmonton, AB, Canada, 24-28 June 2013; pp. 57-61.

19. Yager, R.R. Pythagorean membership grades in multi-criteria decision making. IEEE Trans. Fuzzy Syst. 2014, 22, 958-965. [CrossRef]

20. Yager, R.R. Generalized Orthopair Fuzzy sets. IEEE Trans. Fuzzy Syst. 2017, 25, 1220-1230. [CrossRef]

21. Yager, R.R. On ordered weighted averaging aggregation operators in multicriteria decisionmaking. IEEE Trans. Syst. Man, Cybern. 1988, 18, 183-190. [CrossRef]

22. Xu, Z.S. Intuitionistic fuzzy aggregation operators. IEEE Trans Fuzzy Syst. 2007, 15,1179-1187.

23. Zhang, X.L.; Xu, Z.S. Extension of TOPSIS to multi-criteria decision making with Pythagorean fuzzy sets. Int. J. Intell. Syst. 2014, 29, 1061-1078. [CrossRef]

24. Garg, H.; Arora, R. A nonlinear-programming methodology for multi-attribute decision-making problem with interval-valued intuitionistic fuzzy soft sets information. Appl. Intell. 2018, 48, 2031-2046. [CrossRef]

25. Nancy, G.H. Linguistic single-valued neutrosophic prioritized aggregation operators and their applications to multiple-attribute group decision-making. J. Ambient. Intell. Humaniz. Comput. 2018, 9, 1975-1997.

26. Dombi, J. A general class of fuzzy operators, the DeMorgan class of fuzzy operators and fuzziness measures induced by fuzzy operators. Fuzzy Sets Syst. 1982, 8, 149-163. [CrossRef]

27. Ashraf, S.; Abdullah, S.; Mahmood, T. Spherical fuzzy Dombi aggregation operators and their application in group decision making problems. J. Ambient. Intell. Humaniz. Comput. 2019, 11, 2731-2749. [CrossRef]

28. Akram, M.; Yaqoob, N.; Ali, G.; Chammam, W. Extensions of Dombi Aggregation Operators for Decision Making under m-Polar Fuzzy Information. J. Math. 2020, 6, 1-20. [CrossRef]

29. Du, W.S. More on Dombi operations and Dombi aggregation operators for q-rung orthopair fuzzy values. J. Intell. Fuzzy Syst. 2020, 39, 3715-3735. [CrossRef]

30. Jana, C.; Senapati, T.; Pal, M.; Yager, R.R. Picture fuzzy Dombi aggregation operators: Application to MADM process. Appl. Soft Comput. J. 2019, 74, 99-109. [CrossRef]

31. Lu, X.; Ye, J. Dombi aggregation operators of linguistic cubic variables for multiple attribute decision making. Information (Switzerland) 2018, 9. [CrossRef]

32. Shi, L.; Ye, J. Dombi aggregation operators of neutrosophic cubic sets for multiple attribute decision-making. Algorithms 2018, 11, $1-15$.

33. Chen, J.; Li, S.; Ma, S.; Wang, X. m-Polar Fuzzy Sets: An Extension of Bipolar Fuzzy Sets. Sci. World J. 2014. [CrossRef]

34. Jun, Y.B.; Kim, C.S.; Yang, K.O. Cubic Sets. Ann. Fuzzy Math. Informatics 2012, 4, 83-98.

35. Riaz, M.; Hashmi, M.R. MAGDM for agribusiness in the environment of various cubic m-polar fuzzy averaging aggregation operators. J. Intell. Fuzzy Syst. 2019, 37, 3671-3691. [CrossRef]

36. Riaz, M.; Hashmi, M.R. Linear Diophantine fuzzy set and its applications towards multi-attribute decision making problems. J. Intell. Fuzzy Syst. 2019, 37, 5417-5439. [CrossRef]

37. Riaz, M.; Kalsoom, M.R.H.H.; Pamucar, D.; Chu, Y. Linear Diophantine fuzzy soft rough sets for the selection of sustainable material handling equipment. Symmetry 2020, 12, 1-39. [CrossRef] 
38. Riaz, M.; Hashmi, M.R.; Pamucar, D.; Chu, Y.M. Spherical linear Diophantine fuzzy sets with modeling uncertainties in MCDM. Comput. Model. Eng. Sci. 2021, 126, 1125-1164.

39. Kamaci, H. Linear Diophantine fuzzy algebraic structures. J. Ambient. Intell. Humaniz. Comput. 2021. [CrossRef]

40. Karaaslan, F. Neutrosophic soft set with applications in decision making. Int. J. Inf. Sci. Intell. Syst. 2015, 4, 1-20.

41. Liu, P.; Ali, Z.; Mahmood, T.; Hassan, N. Group decision-making using complex q-rung orthopair fuzzy Bonferroni mean. Int. J. Comput. Intell. Syst. 2020, 13, 822-851. [CrossRef]

42. Liu, P.; Wang, P. Multiple attribute group decision making method based on intuitionistic fuzzy Einstein interactive operations. Int. J. Fuzzy Syst. 2020, 22, 790-809. [CrossRef]

43. Liu, X.; Ju, Y.; Yang, S. Hesitant intuitionistic fuzzy linguistic aggregation operators and their applications to multi-attribute decision making. J. Intell. Fuzzy Syst. 2014, 26, 1187-1201. [CrossRef]

44. Jain, A.; Darbari, J.; Kaul, A.; Jha, P.C. Selection of a green marketing strategy using MCDM under fuzzy environment. In Soft Computing for Problem Solving; Springer: Berlin/Heidelberg, Germany, 2020; doi:10.1007/978-981-15-0184-5_43. [CrossRef] 